\title{
The phylogenomic landscape of the genus Serratia
}

David J. Williams ${ }^{1,2}$, Patrick A. D. Grimont ${ }^{3}$, Adrián Cazares $^{2,4}$, Francine Grimont ${ }^{3}$, Elisabeth Ageron $^{3}$, Kerry A. Pettigrew ${ }^{5}$, Daniel Cazares ${ }^{2}$, Elisabeth $\mathrm{Njamkepo}^{6}$, François-Xavier Weill ${ }^{6}$, Eva Heinz $^{2,7}$, Matthew T. G. Holden ${ }^{5}$, Nicholas R. Thomson ${ }^{2,8 *}$ and Sarah J. Coulthurst ${ }^{1 *}$.

${ }^{1}$ Division of Molecular Microbiology, School of Life Sciences, University of Dundee, Dundee, UK. ${ }^{2}$ Wellcome Sanger Institute, Wellcome Genome Campus, Hinxton, UK. ${ }^{3}$ Unité Biodiversité des Bactéries Pathogènes Emergentes, INSERM Unité 389, Institut Pasteur, Paris, France. ${ }^{4}$ European Bioinformatics Institute, Wellcome Genome Campus, Hinxton, UK. ${ }^{5}$ School of Medicine, University of St Andrews, St Andrews, UK. ' Institut Pasteur, Université de Paris, Unité des Bactéries Pathogènes Entériques, Paris, France. ${ }^{7}$ Departments of Vector Biology and Clinical Sciences, Liverpool School of Tropical Medicine, Liverpool, UK. ${ }^{8}$ Department of Infection Biology, London School of Hygiene and Tropical Medicine, London, UK.

*Correspondence may be addressed to Nicholas Thomson (nrt@sanger.ac.uk) or Sarah Coulthurst (s.j.coulthurst@dundee.ac.uk) 
bioRxiv preprint doi: https://doi.org/10.1101/2022.01.11.475790; this version posted January $11,2022$. The copyright holder for this preprint (which was not certified by peer review) is the author/funder, who has granted bioRxiv a license to display the preprint in perpetuity. It is made available under aCC-BY 4.0 International license.

\section{$1 \quad$ Abstract}

2 The genus Serratia has been studied for over a century and includes clinically-important and diverse 3 environmental members. Despite this, there is a paucity of genomic information across the genus and a

4 robust whole genome-based phylogenetic framework is lacking. Here, we have assembled and analysed 5 a representative set of 664 genomes from across the genus, including 215 historic isolates originally 6 used in defining the genus. Phylogenomic analysis of the genus reveals a clearly-defined population 7 structure which displays deep divisions and aligns with ecological niche, as well as striking congruence 8 between historical biochemical phenotyping data and contemporary genomics data. We show that 9 Serratia is a diverse genus which displays striking plasticity and ability to adapt to its environment, 10 including a highly-varied portfolio of plasmids, and provide evidence of different patterns of gene flow 11 across the genus. This work provides an essential platform for understanding the emergence of clinical 12 and other lineages of Serratia. 
The genus Serratia was originally described in Italy in the early $19^{\text {th }}$ century, following the observation of a blood-like red discoloration appearing on polenta from organic growth ${ }^{1}$. It has since become clear that Serratia species are ubiquitous, free-living, motile Gram-negative proteobacteria, traditionally considered members of the Enterobacteriaceae. The genus Serratia represents a broad and diverse genus of more than ten species, delineated by DNA-DNA hybridization and characterized by extensive physiological and biochemical tests ${ }^{2-12}$. Despite being a diverse genus, much of the contemporary research and understanding of Serratia has focused on the type species, Serratia marcescens. S. marcescens has served as a model system for studying key bacterial traits, including protein secretion systems ${ }^{13}$ and motility ${ }^{14}$, but it also represents an important opportunistic human pathogen ${ }^{15-17}$ for which there has been a dramatic rise in the incidence of multi-drug resistance and reported cases of problematic nosocomial infections ${ }^{18}$.

Other members of this genus include S. rubidaea and S. liquefaciens, which have also been reported to cause hospital-acquired infections, albeit less frequently ${ }^{17,19-21}$. In addition to infection of human hosts, members of multiple Serratia species represent insect pathogens or are otherwise associated with insects. Serratia entomophila has been used as a biocontrol agent in New Zealand to predate upon the pasture pest, Costelytra zealandica ${ }^{12,22-25}$, and S. proteamaculans ${ }^{26}$ and S. marcescens ${ }^{13,27-29}$ have also been shown to be insect pathogens. In contrast, S. ficaria is associated with the pollination and oviposition cycle between figs and fig wasps, respectively ${ }^{6}$. In addition, underlining the ubiquitous nature of this genus, Serratia species can be found in a multitude of environmental niches ${ }^{7,9,29-36}$, including frequent isolation from aqueous environments ${ }^{17,21}$.

Given its importance to human health, it is perhaps unsurprising that the majority of genomic information available for Serratia originate from clinically-derived S. marcescens. Recently a number of $S$. marcescens sequences have also been included within large scale metagenomic studies from preterm neonates ${ }^{90}$ or nosocomial environments ${ }^{91}$. However, for these, as for all the sequences from clinically isolated strains, there is a critical lack of a robust phylogenetic framework for the Serratia genus within which the $S$. marcescens sequences can be placed. In an attempt to understand the many facets and functions of the different species within the Serratia genus, and, importantly, to understand the context within which $S$. marcescens is becoming more widespread as a problematic opportunistic pathogen, we aimed to assemble a balanced genomic dataset that reflected the entire Serratia genus.

42 We supplemented existing publicly-available, published Serratia genome sequences by sequencing Serratia isolates that were non-clinical in origin, and mainly belonging to Serratia species other than S. marcescens. We included the historic collection of Patrick Grimont, located at the Institut Pasteur, a collection that includes the original strains used to define biochemically and phenotypically the vast majority of the known Serratia species ${ }^{3,4,6,9-12}$ These strains, kept in cold storage for between 20 to 40 47 years, represented a unique resource, providing the opportunity to compare historical biochemical, 
together all of the previous molecular and biochemical knowledge from this important genus and place it in a genomics framework. This not only explains why previous definitions of this genus were robust, or not, it also highlights important differences in diversity, plasticity and niche adaptation of the species within it.

\section{Results}

\section{Deep divisions demarcate phylogroups within Serratia}

Here we sequenced and analysed a collection of 256 novel Serratia genome sequences and combined these data with 408 published genomes. Our total set of 664 genome sequences included those of 215 isolates from the original genus-defining Grimont collection sequenced here, 205 multidrug-resistant $S$. marcescens isolates from UK hospitals isolated between 2001 and $2011^{18}$, and an additional 41, more diverse, Serratia isolates from UK hospitals, sequenced here for comparison with the latter collection ${ }^{18}$.

We inferred the genus phylogeny from the whole genome data using a core-gene alignment-based approach. It is evident from Figure 1 that there are deep divisions within this phylogeny that correlate with both the current genus taxonomy and with species-level grouping calculated using genome-wide average nucleotide identity (ANI; clustered using a cutoff of 95 percent; Fig. 1, Supplementary Fig. 2). To gain a finer-scaled view, we used hierarchical Bayesian clustering (FastBaps) to four levels in order to further subdivide the phylogeny and the species-level groups. In total, we identified 7, 16, 23 and 27 clusters across the four levels, respectively (Supplementary Fig. 3). FastBaps level 1 clusters comprise monophyletic clades reflecting individual or multiple ANI groupings within the genus, consistent with speciation or species complexes ${ }^{37}$. The second and third levels reveal the presence of several subdivisions within some of the species-level phylogroups (Fig. 1, Supplementary Fig. 3). Hereafter, we refer to the clusters set out by FastBaps level 3 as Lineages 1-23 (L1-23; Fig. 1).

Interestingly, within the tree there are two examples of singleton genomes occupying both a single ANI species-level phylogroup and representing a discrete FastBaps lineage (L16 and L23). Although both are situated within well-defined species, these two singletons are hereafter referred to as " $S$. marcescens-like" and "S. rubidaea-like", for L16 and L23, respectively. Our phylogeny also resolves previous taxonomic discrepancies. Here, based on the core-gene phylogeny, the liquefaciens complex, made up of S. liquefaciens, S. grimesii, and S. proteamaculans, is monophyletic (Fig. 1). Previous work had suggested $S$. proteamaculans be resolved into both $S$. proteamaculans sensu stricto and a subspecies, termed S. proteamaculans subs. quinovora ${ }^{10,21}$. However, a species level distinction between these two taxa, rather than a sub-species one, was subsequently proposed ${ }^{30}$. We observe that genomes labelled as $S$. proteamaculans and S. proteamaculans subs. quinovora form two separate ANI phylogroups in a monophyletic branch made up of L6-8 (Fig. 1, Supplementary Fig. 2). This supports 
the presence of two distinct species-level groups, which we refer to as $S$. proteamaculans and $S$. quinivorans in accordance with the latter work ${ }^{30}$. Furthermore, only a single genome links L7 and L8 into a single ANI phylogroup within S. quinivorans (Supplementary Fig. 4), which may suggest that a further sub-species separation within S. quinivorans is appropriate.

\section{Concordance between historical biochemical phenotyping and metabolomic predictions}

The current taxonomic structure of the Serratia genus, summarised by Grimont and Grimont, $2005{ }^{92}$, is based on 41 phenotypic and biochemical tests used to differentiate between different Serratia species or species-complexes. Many of the Serratia isolates originally used to define the genus taxonomy were sequenced here (Fig. 1; Supplementary Table 1), presenting a unique opportunity to reconcile this historical biochemical metadata with genomic predictions. First, we calculated the genus pan-genome using a population structure-aware approach ${ }^{38}$. The pan-genome comprised 47,743 discrete gene groups (Fig. 2a), of which 2252 were present in at least 99 percent of all genomes in the dataset (which would be defined as a "traditional" core genome), however only 1655 of these were present in at least 95 percent of genomes within each FastBaps lineage, and therefore are core to all lineages (Fig. 2b). These 1655 genes are hereafter defined as the "genus-core". From the 47,743 genes of the pan-genome, we predicted the metabolic potential of the genus. We identified 641 different complete metabolic pathways using Pathway tools ${ }^{39}$ (Fig. 3a), of which 260 were core to the genus, being present in all known lineages (L1-23) (Fig. 3a, Supplementary Fig. 9).

Of the 41 metabolic tests used to define species within the genus, some were also used to define biotypes within a species. It can be seen that the fine-scale delimitation of biotypes within the liquefaciens complex corresponds with the phylogenetic structure we observe (Supplementary Fig. 10). Similarly, ten of the 41 metabolic tests were used previously to split marcescens into ten biotypes, which reflected differences in niche occupancy ${ }^{21}$. It is clear that these biotypes are also robust markers of phylogenetic subdivisions within this important species (Fig. 3c, 4c).

In silico pathway predictions were used to identify the genes and/or pathways linked to the biotype tests for $S$. marcescens, where strain biotype metadata was available. Four of these ten tests (growth on $m$ erythritol, trigonelline, 3-hydroxybenzoate and lactose) were not investigated because there were no corresponding pathway assignments in the predicted metabolic network. Next, the phylogenetic distribution of these metabolic genes was plotted across the phylogeny and extrapolated back to the most basal internal node differentiating two biotypes, based on gene/pathway presence or absence (Fig. $3 b)$. Where we had no genome representative of a particular biotype in which to identify the genes for the cognate pathway, these were predicted using the results of the in silico metabolic prediction for known pathways (Fig. 3c). Although there are some discrepancies between pathway presence/absence and historical phenotypes for different biotypes, Fig. 3b shows a robust linkage between inferred phylogeny and biotype. Across the species, these data show that L15 corresponds with the pigmented 
biogroups A2, A6 and A1, whilst L14, L12 and L9 correspond with the nonpigmented biogroup A4 and biotypes TCT and A5. Furthermore, adding the source of sample isolation shows that niche occupancy also aligns well with the population structure and biotype data (Fig. 3c). In particular, strains representing original biotypes described in the 1980s that were associated with hospitalised patients

122 (TCT, TC, TT, A5, A8) are situated in the same phylogenetic position as contemporary clinically-

123 isolated $S$. marcescens, implying important adaptations that can be linked to risk of disease or greater

124 fitness in hospital environments.

\section{Codon usage redundancy may facilitate a GC shift within Serratia}

126 Changes in GC content of coding sequences over time have been hypothesised to reflect subtle 127 differences in mutational bias as a consequence of long-term niche adaptation or different lifestyles ${ }^{40}$.

128 Given that there are clear differences in the lifestyles and niches between Serratia species and intra129 species lineages, we investigated the distribution of GC content across the genus. We observe that 130 Serratia is broadly divided into two phylogenetically-coherent groups based on whole-genome GC 131 content: marcescens, entomophila, ficaria and rubidaea show a GC content of $\sim 59 \%(59.0-59.9 \%)$, 132 whilst odorifera, fonticola, plymuthica, liquefaciens, proteamaculans, quinivorans and grimesii have a 133 GC content ranging from 52.7 to $56.1 \%$ (Fig. 4a). The singleton "S. rubidaea-like" and "S. marcescens134 like" genomes have an average GC content of 57.7\% and 58.9\%, respectively, consistent with their 135 positions in the tree adjacent to rubidaea or marcescens. Additionally, we observed no difference in 136 overall GC content between coding and non-coding regions (Fig. 4b).

137 To understand how this GC pattern impacts on protein coding, we investigated the variation in GC 138 content over the three codon positions for all lineages using the genus-core set of 1655 genes (Fig. 4c; 139 Fig. 2b), and separately for all other genes, designated "non-core". We observed no obvious difference 140 in GC content between genes that were core or non-core at all three codon positions, termed as GC1-3 141 (Fig. 4c). The GC content at GC2 is essentially fixed across the genus (Fig. 4c), whilst GC1 shows a 142 slight skew across the genus, varying by approximately 1\%. Codon position GC3 showed a clear bias 143 for A/T-ending codons in low GC species and G/C-ending codons in high GC species, as expected ${ }^{41}$ 144 (Fig. 4d). Hence, the difference in average G/C across the genus is largely explained by variation in 145 codon position GC3. For example, GC3 in S. grimesii is 20\% lower than in S. marcescens L9 (Fig. 4c).

146 Taken together, the variations in metabolic capability and GC content between both species and niche147 adapted lineages are indicative of long-term niche adaptation within evolutionary timescales.

\section{Pan-genome analysis highlights lineage-specific gene gain and loss as well as intra-genus gene}

\section{9 flow}

150 The results so far suggest that the pan-genome of Serratia lineages is phylogenetically constrained, yet 151 members of Enterobacteriaceae are known to have a highly plastic gene content through horizontal 
gene transfer (HGT). To investigate this further, we sought to understand genus-wide species plasticity. Plasticity can be estimated by comparing the pan-genome size and complexity against the size of the genus-core gene set (Fig. 2a, b). Given the uneven sampling of some taxa we performed a population structure-aware analysis of the pan-genome, as noted above, in order to define the "genus-core" genome. We overlaid this classification system ${ }^{38}$ onto intersections of multi- and single-lineage core genomes (Fig. 2b). Genes were defined as core to a lineage if a gene was present in at least 95 percent of the genomes in each lineage, and the union of all lineage-core genes was defined as the genus-core, consisting of 1655 genes (Fig. 2a,b). This analysis showed lineage- and species-level core gene gain and loss, which are markedly larger in terms of the number of genes when looking at lineages that have a very small sample size. For example, $S$. odorifera (L21; two genomes) and S. marcescens-like (L16; one genome), have 1725 and 345 genes core only to those specific lineages (Fig. 2b).

Significant variations in the pan-genome between different Serratia species were evident. For example, whilst $S$. entomophila and $S$. fonticola display similar core gene branch lengths, indicative of similar evolutionary timescales, S. entomophila has a closed pan genome whilst $S$. fonticola has an open pangenome (Fig. 2c). The difference in the size of the accessory genome between these two species is 6395 genes, with S. entomophila and S. fonticola and having accessory genome sizes of 2764 and 9159 genes, respectively (Supplementary Table 2). In contrast, S. ficaria, which has similar internal branch lengths, has a more open pan-genome curve (Fig. 2c), and an accessory genome of 3490 genes, despite being represented by fewer genomes in the analysis (Supplementary Table 2), suggesting that different Serratia species have varying propensities to gene gain and loss.

Evidence of core gene gain and loss possibly reflective of speciation or niche adaptation can be seen when examining this data. For example, 99 genes are found core to all three lineages in S. entomophila, and 41 genes are found core to the entire S. liquefaciens complex, which comprises S. liquefaciens, grimesii, proteamaculans and quinivorans (Fig. 2b). Within the pan-genome we identified lineage- and species-exclusive gene sets, as well as those whose genes are also present at intermediate or rare frequencies across the genus (Fig. 2b). For example, of the 99 S. entomophila species-core genes, 35 genes were found across the rest of the genus (Supplementary Fig. 5), shared between both high and low GC members. In contrast, in the 41 genes core to the $S$. liquefaciens complex, very few are found outside the complex, and where they are, they are predominantly present in S. ficaria and S. plymuthica (Supplementary Fig. 6). The sharing of genes across the genus, implying potential gene flow, raises questions about whether GC3 has been ameliorated to reflect the GC3 trend in a potential recipient genome. Of the 35 genes from the high GC species $S$. entomophila that are found across the low GC species S. liquefaciens complex, S. plymuthica and S. fonticola, the GC3 values of these genes are lower than when found in S. entomophila (Supplementary Fig. 11). Similarly, S. liquefaciens complex core genes which are also found in S. ficaria and S. plymuthica, both species with higher GC than members of the liquefaciens complex, appear to have ameliorated GC3 (Supplementary Fig. 12). 
In an attempt to understand the mechanisms by which genes are gained and lost, we focused initially on $S$. marcescens. We investigated the genetic context of the metabolic gene loci associated with different biotypes. In doing so, we identified a hypervariable locus analogous to the plasticity zone seen in Yersinia ${ }^{42}$. Variation in this locus explained some of the biochemical differences seen within $S$. marcescens. This plasticity zone was located between two sets of tRNAs: one encoding tRNA-Proggg, the other encoding tRNA-Ser ${ }_{\text {tga }}$ and $\mathrm{tRNA}-\mathrm{Thr}_{\mathrm{tgt}}$. It encoded the genes required for gentisate degradation (nag gene cassette) and/or tetrathionate reduction (ttr gene cassette), present in the same order and orientation across the species, located alongside three sets of genes that were variably present across the $S$. marcescens phylogeny (Fig. 5). These three sets comprised: (1) four genes including one encoding a cyclic AMP (cAMP) phosphodiesterase; (2) an acyltransferase; and (3) a two-gene toxin cassette. A gene predicted to encode a DNA damage inducible protein I ( $\operatorname{din} I)$ was always present, downstream of the ttr/nag/cAMP genes and upstream of the acyltransferase gene. In a small number of instances, frameshifts have truncated or split coding genes in this region. Additionally, prophage sequences can also be found flanking these variable sets of genes in some genomes (Fig. 5). Interestingly, in L13 and L9, when the nag genes are present, an additional gene, encoding a gene with predicted 3chlorobenzoate degradation activity, is present 3' of the other genes in the cassette (Fig. 5).

Further evidence of gene flow can be seen in S. marcescens (Supplementary Figs. 7, 8). Certain genes core to $S$. marcescens L10, L11 and L14 were also found in members of of other lineages, including $S$. marcescens L15 and L9, and S. proteamaculans L6 (Supplementary Fig. 8). On closer inspection, the genes shared with $S$. marcescens L15 and S. proteamaculans L6 comprise a Type VI Secretion System (T6SS). Whilst polyphyletic across $S$. marcescens, this T6SS is syntenic when found in $S$. marcescens but is encoded in a different region of the chromosome when present in S. proteamaculans. In both cases, this T6SS is encoded adjacent to a tRNA, and also an integrase in S. proteamaculans, potentially suggestive of horizontal transfer across the genus from marcescens. There are also 42 genes core to the clinically-associated $S$. marcescens L9, for which 37 are also found polyphyletically across the rest of S. marcescens (Supplementary Fig. 8). Many of these genes are predicted to be components of fimbrial usher systems (Supplementary Fig. 8).

\section{Contribution of plasmids to gene content and flow varies across the Serratia genus}

To understand the potential contribution of plasmids to the plasticity seen in this genus, we searched for plasmid contigs in our genus-wide dataset. This uncovered 409 putative plasmids in 228 genomes and 9 species, 301 (73\%) of them present in S. marcescens (Fig. 6; Supplementary Table 3). The collection of identified plasmids displays a wide range of sizes ( $\sim 1-310 \mathrm{~kb})$ and GC content ( $\sim 30-66 \%)$, indicating diversity. However, the distribution of these traits varied amongst Serratia species (Supplementary Figs. 13-15). For example, plasmids identified in S. marcescens and liquefaciens show a markedly broader range of size and GC content compared with those detected in S. entomophila and 
quinivorans. Seventy out of a total of 113 predicted plasmid replicons were found within $S$. marcescens

224 L9 and L12, which are the 'clinical' lineages in which $97 \%$ and $81 \%$ percent of the isolates, respectively, are known to be human- or clinically-associated. In terms of mobility, 296 (72\%) of the plasmids were predicted to be conjugative or mobilizable (Fig. 6; Supplementary Table 3), highlighting their potential role in HGT. Consistent with this notion, the predicted host range for this collection of plasmids ranges from single genus to multi-phyla, with the most heterogeneous host range profile observed for plasmids found $S$. marcescens (Fig. 6).

A network visualization of the all-versus-all Mash distances ${ }^{43}$ calculated for the Serratia plasmids was used to explore their diversity. The resulting network comprises 113 clusters, of which 53 (47\%) correspond to singletons, illustrating the diversity of Serratia plasmids (Fig. 6). Differences in plasmid abundance between clusters were evident from the network, as four top clusters included $36 \%$ of the plasmids identified in Serratia genomes. Overall, the plasmids clustering was concordant with their size and GC content but also with the host species (Fig. 6c, Supplementary Fig. 15), suggesting limited between-species plasmid transfer within Serratia. Nevertheless, some multi-species clusters were identified, perhaps hinting at recent plasmid acquisition events. A cluster formed by plasmids of four non-marcescens species was the largest in the network. This cluster mainly consists of large MOBP conjugative plasmids related to the amber disease associated plasmid (pADAP), which is required for virulence of $S$. entomophila and $S$. proteamaculans in the larvae of the grass grub Costelytra zealandica $^{12,22}$. Interestingly whilst pathogenic potential in Costelytra zealandica is a defining trait of

242 S. entomophila, the presence of a pADAP-related plasmid was not universal or a defining trait for either

243 S. entomophila or S. proteamaculans, being found in members of S. entomophila, S. quinivorans, and

244 S. proteamaculans, and a single S. liquefaciens genome.

Notably, the predicted host range of the plasmids brings an additional perspective on their potential dynamics within the genus. Most plasmids identified in $S$. marcescens appear to be restricted to this species within Serratia. Yet many of them have a predicted host range that goes beyond the taxonomic rank of family, implying transfer outside the genus, including two clusters of small ColRNAI plasmids predicted to cross multiple phyla (Fig. 6). In contrast, the largest plasmid cluster (pADAP-like), featuring multiple non-marcescens species, seems to be restricted to the Serratia genus. Altogether, this picture may suggest that the ecological niche of $S$. marcescens has favoured plasmid exchange with diverse hosts outside the genus but has also promoted plasmid containment within the species in implies a major role for this species in the gene flow outside the genus and to a lesser but relevant extent within it.

257 A famous characteristic often popularly associated with Serratia spp. is the production of the red 
pigment prodigiosin ${ }^{17}$. However, in fact, prodigiosin production has only been observed in $S$. marcescens biogroups A1, A2 and A6, some rubidaea and some plymuthica isolates ${ }^{21}$. The pig gene cluster comprises fourteen genes (pigA-pigN) required for the production of prodigiosin ${ }^{44}$. Searching across the genus for pig gene cluster loci and flanking regions showed that, consistent with the earlier biotyping observations, the pig cluster is only encoded in certain $S$. marcescens, $S$. rubidaea and $S$. plymuthica genomes (Figs. 3, 7) which are associated with biotypes or biogroups known to be pigmented. In each case, the cluster presents exactly the same contiguous order of genes (pigA-pigN), however, notably, it is found in separate genomic loci in each of the three different species (Fig. 7). Representative pig gene clusters from each species share $\sim 77-80 \%$ identity at the nucleotide level (Fig. $7 \mathrm{~b}$ ), which is similar to the shared nucleotide percentage identity between these species at fully syntenic regions in the chromosome. This indicates that the pig gene cluster has been acquired horizontally on at least three separate occasions.

\section{Discussion}

With advancements in technology, the methods used to delineate and decipher prokaryotic species boundaries have changed over time, as researchers attempt to resolve the shortcomings of earlier approaches and build upon the understanding of biology at any given point in time. This study has, in part, investigated the relationship by which species level boundaries have been determined within a genus, namely phenotypic characterisation and whole genome sequencing. It also highlights how, in order to make appropriate conclusions from these approaches, the currently available data requires to be constantly filtered, checked and reviewed.

Following its original identification in the early 19th century, the nomenclature and number of species within Serratia underwent several iterations as additional strains with similar, yet distinct phenotypes were identified and added to an expanding membership of the genus ${ }^{17,21}$. Then in the 1970s and 80s, comprehensive biochemical and phenotypic characterisation along with the use of DNA-DNA hybridisation, allowed the genus to be defined as a collection of ten clearly defined species. Since the advent of the genomic era, despite the potential of genomic approaches to resolve fine-scaled differences between taxa, no similar-scale work within Serratia has been attempted, nor do we have a robust phylogenetic framework against which we are able to recognize novel Serratia spp or emerging lineages. Such a framework is also required to resolve confusion over existing species. For example, strain DSM 21420, a nematode-associated strain proposed to belong to S. nematodiphila ${ }^{45}$, sits within the broadly non-clinical $S$. marcescens L15, suggesting that it does not in fact represent a separate species. Conversely, the identification of singleton ANI phylogroups and FastBaps lineages $(S$. marcescens-like L16 and S. rubidaea-like L23) highlights that there is likely further species diversity to be discovered. This may be partly due to geography and lack of sampling: the strain that occupies 
L16 (MSU97) was sourced from a plant in the Carrao River in Venezuela ${ }^{46}$, a region which is not highly sampled.

It is interesting to consider how the computational approaches used here to classify and describe the genus parallel the original biotyping. In the earlier studies, in vitro DNA-DNA hybridisation was used to assess genomic relatedness between novel Serratia strains $^{5,11}$, an approach for which ANI is in many ways an in silico proxy, whilst the connection between in silico prediction of metabolic potential and the lab-based tests detecting the corresponding metabolic pathway in the original biochemical-based biotyping is obvious. Furthermore, in some cases these biotypes highlight further clusters within lineages that match branching within the phylogeny. For example, biotypes C1c, EB and RB, and biotypes A1b, A1a, A6 and A2 are all monophyletic within S. proteamaculans L6 and S. marcescens L15, respectively (Figs. 3b, 5). This highlights just how accurate the original biochemical-based typing was for defining species.

This accuracy is particularly striking given that we have observed that presence or absence of metabolic pathways (corresponding to the historic biotyping tests used) can be due to repeated gene gain or loss in the same locus over short evolutionary distances. For example, the genes required for the degradation of gentisate and the reduction of tetrathionate are gained and lost within and between lineages in $S$. marcescens, in the same locus and also in the same conserved order (Fig. 5). This would explain why the original phenogrouped biotypes based on biochemical typing had "variable" results for certain metabolic tests, such as gentisate degradation being observed to be variable in the clinical biotypes A8a and $\mathrm{A} 8 \mathrm{c}^{92}$. This locus-specific pathway gain and loss in historic isolates is also seen in more contemporary strains (Fig. 5). The maintenance of this plasticity zone suggests that there are transient and frequently re-occurring environmental selective pressures where the benefit and cost of these pathways is great enough to provide selection both for and against them. In other words, the data suggest that both the loss and re-acquisition of these elements is of benefit to $S$. marcescens at various times.

It is also noteworthy that the environment from which strains were isolated across our assembled dataset tends to match the environments and niches with which each biotype was historically associated ${ }^{21}$. Of particular interest is the observation that the predominantly hospital-associated biotypes of $S$. marcescens that were defined in the 1980s (A5, A8, TCT) sit within L9 and L12 defined in the current study. These lineages are mainly comprised of recently-sequenced genomes from hospital settings, including a large collection of clinically-derived $S$. marcescens isolates from the UK that represent the recent emergence of hospital-adapted clones exhibiting recent acquisition of MDR phenotypes ${ }^{18}$. The fact that these lineages of clinically-associated S. marcescens were identified back in the 1970s and 80s shows that the original biochemical characterisation of Serratia captured the emergence of $S$. marcescens lineages that have subsequently been reported to be associated with human disease many times in recent years ${ }^{18,19,47-52}$. The apparent specialisation of S. marcescens L9 to be a clinically-adapted 
pathogen is further highlighted by plasmid replicon identification and the types of lineage-specific core genes observed. The identification of numerous plasmid replicons in these lineages (L9, L12 and L14) as opposed to the rest of the genus is perhaps unsurprising, given that most known plasmids are associated with multi-drug resistance and hospital environments. Fimbrial genes are well-known pathogenicity factors and multiple different fimbrial genes are found to be core to L9 but accessory to multiple other $S$. marcescens lineages. This potential gene flow from L9 across the rest of $S$. marcescens may be one reason why isolates from more "environmental" S. marcescens lineages are still isolated from nosocomial settings. In these other lineages, S. marcescens is still an opportunistic pathogen, with nosocomial isolates being genetically similar to strains that have colonised or infected plants, insects or other environments. Indeed, bee-associated $S$. marcescens cause infections in bees in a similar manner to how $S$. marcescens can cause bloodstream infections in preterm neonates ${ }^{29}$. Taking the historic biotyping data along with the population structure defined here, the combined data suggest that $S$. marcescens is highly plastic in its nature yet can also become specialised in a particular niche.

Speciation and niche specialisation events or processes are seen across the phylogeny, as highlighted by the long branch lengths between divisions, separations in GC content, variation in metabolic potential, and enrichment for certain isolation source sites in different lineages. These divisions likely represent ancient speciation events that have occurred as Serratia has spread to be ubiquitous worldwide. As mentioned above, changes in GC content can be a response to long-term niche adaptation, however there is no commonly held theory or understanding of the possible reasons that underpin this. One possible factor that may have influenced the variation in GC content observed across Serratia is a difference in ideal growth temperature: higher GC Serratia species tend to be able to grow better at higher temperatures than lower GC Serratia species ${ }^{21}$. Another possibility is that the observed GC-dependent change in codon usage, which does not alter protein sequence or function, is indicative of a shift to an optimal set of codons for each particular Serratia species, although the evolutionary pressure that would drive such a shift is not clear. Importantly, however, this division in GC content does not seem to be a barrier for gene flow in Serratia, since genes core to the high GC species $S$. entomophila can also be found in polyphyletic and variable patterns across the genus, including in low GC Serratia species (Supplementary Fig. 11). However, it is formally possible that these genes could have been horizontally acquired from non-Serratia sources.

This study also provides definitive genomic evidence to explain the variation in a classical Serratia phenotype, namely the production of the red pigment prodigiosin (Fig. 7). The high level of synteny within the pig gene cluster together with the absence of homology in the flanking regions indicates that the ability to produce prodigiosin has been acquired on at least three separate occasions within Serratia, namely in subsets of $S$. marcescens, S. plymuthica and S. rubidaea. Given the relatively low shared nucleotide identity, it is unclear when and how these genes were incorporated into the chromosome, 
363 and whether each event reflects gene flow within the genus or separate acquisition from an external

364 source. This genomic evidence of separate acquisition of the pig clusters matches the historical metadata

365 noting that S. marcescens, S. plymuthica, and S. rubidaea all variably produce a red pigment ${ }^{21}$.

366 Prodigiosin has been reported to display many functions, including anti-protozoal, anti-fungal, anti-

367 bacterial, immunosuppressive, and anti-cancer activity ${ }^{44}$. The biological advantage for these individual

368 Serratia species, or subsets thereof, to be able to produce prodigiosin is unclear, however it could reflect

369 a degree of convergent evolution within Serratia, or perhaps the varied potential functions of

370 prodigiosin may provide different fitness benefits to different species. Further evidence for convergent

371 evolution in the genus is provided by the observation that members of both $S$. proteamaculans and $S$.

372 entomophila carry pADAP, which is required for the pathogenesis of grass grub larvae ${ }^{22,53}$.

373 In conclusion, we have demonstrated the power of combining phenotypic metadata with a

374 comprehensive and balanced genomics-based phylogeny to define an important and diverse bacterial

375 genus, its plasticity and its niche adaptation. The dataset and phylogeny that we present here will

376 provide a vital platform for future work, including in the tracking of further emergence of pathogenic

377 Serratia or changes in the portfolio of anti-microbial resistance genes or pathogenicity factors. 


\section{Material and Methods}

\section{Bacterial strains}

380 Bacterial isolates sequenced in this study are listed in Supplementary Table 1, along with relevant metadata and summaries of sequencing and assembly statistics.

\section{Bacterial culture and resuscitation, genomic DNA isolation and sequencing}

383

384

385

386

387

388

389

390

391

392

393

394

395

396

397

398

399

400

401

402

403

404

405

406

407

408

409

410

411

412

279 isolates in the Institut Pasteur collection were successfully resuscitated from agar stabs kept in cold storage for $\sim 20$ years. Isolates were resuscitated in the original agar stabs with 2-3 ml of Tryptic Soy Broth and incubated static and upright at $30^{\circ} \mathrm{C}$ for up to three days, or until clear signs of growth were visible, followed by sub-culture on solid LB media. In rare cases of mixed colony morphology, or abnormal looking colonies, a number of colonies were selected and streaked through two to three times. In such cases, the Serratia were identified, where possible, by red pigmentation and/or a strong potatolike odour. In cases of mixed pigmentation, a representative colony of each type of pigment type (or lack of pigment) were taken forward. DNA extraction was carried out using the Maxwell 16 Cell DNA purification kit (Promega) on the automated Maxwell 16 MDx instrument (Promega), according to the manufacturer's instructions. $400 \mu \mathrm{l}$ of mid-log culture (grown at $30^{\circ} \mathrm{C}$ in $\mathrm{LB}$ ), sub-cultured from a liquid overnight culture, was used for DNA extraction. DNA samples were sequenced using the Illumina HiSeq X10 platform (Illumina, Inc) at the Wellcome Sanger Institute. DNA fragments of approximately $450 \mathrm{bp}$ were produced from $0.5 \mu \mathrm{g}$ DNA for Illumina library creation and were sequenced on a $150 \mathrm{bp}$ paired-end run.

42 isolates from UK hospitals were received from frozen stocks, freshly streaked plates, or in bead suspensions, and were grown on solid media to ensure uniform single colonies. As with isolates from the Pasteur collection, samples from mid-log cultures were used for DNA extraction. DNA samples were sequenced using short-read technology only, or a hybrid approach of both long-read and shortread technology, as detailed in Supplementary Table 2. For short-read sequencing, DNA was extracted using a DNeasy extraction kit (Qiagen). DNA quality was assessed using a Qubit 3.0 (Invitrogen) and Bioanalyzer (Agilent), then subsequently diluted to a concentration of $0.4 \mathrm{ng} / \mu 1$. DNA library preparation was performed using the Illumina Nextera protocol and PCR clean up was performed using AMPure beads (Beckman). Multiplexed samples were then run on the MiSeq (Illumina). Adaptor sequences were automatically trimmed by the MiSeq platform and then raw reads were downloaded from basespace in FASTQ format. For long-read sequencing, high molecular weight DNA was isolated using the MasterPure DNA Purification kit (Epicentre, no. MC85200). Sequencing was performed using the PacBio Sequel (Pacific Biosciences) or MinION (Oxford Nanopore Technologies) sequencing platforms. For PacBio sequencing, $10 \mu \mathrm{g}$ DNA was sequenced using polymerase version P6 and C4 sequencing chemistry reagents. For MinION sequencing, $5 \mu \mathrm{g}$ DNA in $35 \mu 1$ nuclease-free water for each sample was sequenced using the SQK-LSK108 kit using a FLO-MIN106 flow cell. DNA ends 
413 were repaired and dA-tailed using NEBNext End Repair/dA-tailing module, following by ligation of 414 barcodes. DNA concentration and clean up steps were performed using AMPureXP beads (New 415 England Biolabs). 12 samples (from 12 isolates) were multiplexed on a single MinION run. Basecalling 416 and demultiplexing was performed by Albacore v2. In all cases, kits were used according to the 417 manufacturers' instructions.

\section{Sequence data quality control}

419 Read sets obtained from all samples were compared to the MiniKraken database by Kraken v0.10 ${ }^{54}$, 420 and then corrected using Bracken ${ }^{55}$ which assigns reads to a specific reference sequence, species or 421 genus. If reads were not able to be assigned to a taxonomic class, they were classed as 'unclassified'.

422 Any read sets that belonged to genera other than Serratia were discarded from any further analysis, 423 along with any assemblies obtained from those read sets.

424 Any read sets with more than an estimated five percent of heterozygous SNPs across the whole genome 425 were removed from further analysis, in addition to any assemblies obtained from those read sets. 426 Heterozygous SNPs were calculated using a software pipeline from the pathogen informatics team at the Wellcome Sanger Institute. Specifically, read sets from each Serratia sample were aligned to an appropriate reference for that sample, given the taxonomic profile from the Kraken and Bracken output.

429 Reads were aligned to the reference using bwa v0.7.17 $7^{56}$, and parsed using samtools $v 0.1 .19^{57}$ and 430 bcftools v0.1.1957. Reads were considered as heterozygous if there were at least two variants at the same 431 base, both supported by a number of reads that was fewer than 90 percent of the total reads mapped to 432 that site. Read coverage to each strand was considered independently. The minimum total coverage 433 required was $4 \mathrm{x}$, and the minimum total coverage for each strand was $2 \mathrm{x}$. Calculated heterozygous SNP 434 coverage was then predicted by scaling the number of observed heterozygous SNPs against the proportion of the reference that was covered by read mapping.

436 Eight genome sequences from the Pasteur collection dataset and one from the UK hospitals set were 437 removed due to the above criteria. In addition, a number of the isolates resuscitated from the Pasteur 438 collection were duplicate samples of the same strain. After inspection of preliminary phylogenetic trees 439 from core-gene alignments (see below), a further 56 genomes were removed from the Pasteur collection 440 dataset due to being duplicates of the same-named strain.

\section{Publicly available genome sequences}

442 Previously-published, publicly-available assembled genome sequences were downloaded from the 443 NCBI GenBank database (https://ftp.ncbi.nlm.nih.gov/genomes/genbank/) as of 19/03/2019. Genomes 444 were downloaded if the species was attributed to any of the following: Serratia sp., odorifera, rubidaea, 445 plymuthica, liquefaciens, grimesii, oryzae, proteamaculans, quinivorans, nematodiphila, ficaria, 446 entomophila or marcescens. Assemblies smaller than 4.5 Mbp or larger than $6.5 \mathrm{Mbp}$ were removed 
from the analysis, along with any assemblies comprised of more than 250 contigs. Quast v4.6.0 $0^{58}$ was used to extract statistics for genomes and genomic assemblies, specifically whole genome GC content, number of contigs and assembly size. Initial phylogenetic trees with additional non-Serratia reference sequences (Yersinia enterocolitica, Rahnella aquatilis and Dickeya solani) were computed, and genomes detemined by visual inspection as being non-Serratia or close to non-Serratia members of Enterobacteriacaeae were removed from any subsequent analysis. Ten genomes were excluded on this basis, including several so-called Serratia sp. and Serratia oryzae.

\section{Genome assembly and annotation}

455

456

457

458

459

460

461

462

463

464

465

466

467

468

469

470

471

472

\section{3}

474

475

476

477

478

479

480

The assembly method used for genome assembly and annotation for each genome are detailed in Supplementary Table 1. For samples sequenced using short-read only data, genomes were assembled in two different ways depending on their origin. Isolates in the Institut Pasteur collection were assembled through assembly pipelines at the Wellcome Sanger Institute. For each sample, sequence reads were used to create multiple assemblies using VelvetOptimiser v2.2.5 (https://github.com/tseemann/VelvetOptimiser) and Velvet v1.259. An assembly improvement step was applied to the assembly with the best N50 and contigs were scaffolded using SSPACE ${ }^{60}$ and sequence gaps filled using GapFiller ${ }^{61}$. For isolates from UK hospitals that were only sequenced by short-read technology, these short reads were assembled using SPAdes v3.6. $1^{62}$, using default settings.

For hybrid short- and long-read assemblies of selected isolates from UK hospitals, genomes were assembled using Unicycler v0.4. $7^{63}$. Long-read-only assemblies from MinION or PacBio long reads were generated first, using Canu v1.6 $6^{64}$, with the expected genome size set as $5.4 \mathrm{Mbps}$, the minimum read length and overlap length set to $100 \mathrm{bp}$, and "corOutCoverage" set to 1000. Long-read assemblies were then used as input to Unicycler, using the --existing_long_read_assembly flag. Sets of paired-end Illumina reads were then used as input to Unicycler alongside this long-read assembly and also the long reads. The "--mode" flag was set to "normal". In the event that Unicycler was not able to produce circularised assemblies, Circlator v1.5.5 $5^{65}$ was used to circularise assemblies.

Assembled genomes were then annotated using Prokka v.1.13.366.

\section{Pan-genome analysis}

Pan-genomes were calculated from 664 Serratia sequences using Panaroo v1.2.367, with Prokkaannotated genomes as input. For initial protein clustering, a protein similarity threshold was set at 95 percent (0.95). The subsequent clustering of these groups into protein families was performed using a threshold of 70 percent identity (0.7). The "--clean-mode" flag was set to "moderate". A core-gene alignment was created using the "-a" flag, specifying mafft as the aligner using the "--aligner" flag, with core genes specified by being present in at least 95 percent of genomes (631/664). Pan-genome gene accumulation curves were generated using the specaccum function from the $\mathrm{R}$ package Vegan 
v2.5. $7^{68}$, with 100 random permutations.

482

483

484

485

486

487

488

489

490

491

492

493

494

495

496

497

498

499

500

501

502

503

504

505

506

507

508

509

510

511

512

513

514

Population structure-aware classification of genes across the genus was performed upon the gene presence/absence matrix created by Panaroo through the use of the twilight analysis package ${ }^{38}$. Groups were defined by the lineages set by the third level of Fastbaps clustering (see below), and singleton lineages were included in the analysis ("'--min_size 1"). The core and rare thresholds were set at 0.95 and 0.15 , respectively.

Preliminary core-gene alignments using the pan-genome software Roary v3.12.069, including all downloaded genomes from the NCBI GenBank datbase, duplicate genomes from the Pasteur collection and non-Serratia Enterobacteriacaeae members, were computed for initial tree-drawing to remove contaminants and assess whether duplicate strains (from data supplied in strain name information, for example, labels on agar stabs from strains in the Pasteur collection) were found in the same position in the tree. Non-Serratia Enterobacteriacaeae were also used to determine the location of the root for all visualisations of the Serratia genus phylogenetic tree.

\section{Clustering, phylogroup determination, core-gene alignment filtering and phylogenetic tree construction}

For the Serratia phylogeny, a concatenated core-gene alignment from 2252 genes $(2,820,212$ bp in length) from Panaroo v1.2.3 ${ }^{67}$ (as described above) was filtered to remove monomorphic sites that were exclusively A, T, G or C using SNP-sites v2.5.170. The resulting alignment was 398,551 bp in length. IQtree v.1.6.10 ${ }^{71}$ was then used for maximum-likelihood tree construction using 1000 ultrafast bootstraps ${ }^{72}$ using the TIM2e + ASC + R4 model chosen using modelfinder ${ }^{73}$. Both the ultrafast bootstraps and modelfinder were implemented in IQtree. The Serratia phylogenetic tree was rooted at the position of a Yersinia enterocolitica outgroup root after analysis of preliminary trees based on exclusively polymorphic variant sites (filtered using SNP-sites v2.4.1) from preliminary core-gene alignments (determined using Roary v.3.12.0 as described above). Trees were constructed using modelfinder implemented in IQtree v1.6.10, followed by tree construction using IQtree v.1.6.10.

Whole-genome assemblies were compared in a pairwise manner using fastANI v1. $3^{74}$, and phylogroups determined through clustering these comparisons using a cutoff of $95 \%$ average nucleotide identity (ANI). Genomic assemblies were then clustered base on this cutoff value, using the script fastANI_to_clusters.py which uses the networkx package (https://networkx.github.io/), and visualised using Cytoscape v3.7.1 ${ }^{75}$. The phylogeny was partitioned into lineages defined through hierarchical bayesian clustering using Fastbaps v1.0.4 ${ }^{76}$. Fastbaps was used to cluster the phylogeny over four levels, with the third levels selected for lineage designation. The SNP sites-filtered core-gene alignment was used as input to Fastbaps, alongside the rooted phylogenetic tree to provide a guide for the hierarchical partitioning. 


\section{Functional and metabolic pathway analysis}

516 In silico reconstruction of metabolic pathways was performed using Pathway tools v $23.5^{39}$, using a 517 multi-processing wrapper tool mpwt (https://github.com/AuReMe/mpwt) ${ }^{77}$. In order to arrange input 518 data into the appropriate format, and subsequently parse the output, a collection of Python and R scripts 519 were written (https://github.com/djw533/pathwaytools_gff2gbk). Further specific information about

520 how to run this can be found in the readme hosted at the github repository. In brief: Representative 521 protein sequences for each of the 47,743 protein family groups identified in the pan-genome analysis 522 were extracted from the pan-genome graph-associated data using Cytoscape v3.7.1, and functionally 523 annotated using EggNOG-mapper v1.0.3 ${ }^{78}$, using the following flags "-m diamond -d none --tax_scope 524 auto --go_evidence non-electronic --target_orthologs all --seed_ortholog_evalue 0.001 -525 seed_ortholog_score 60 --query-cover 20 --subject-cover 0 --override”. Using the EggNOG annotations 526 from representative protein sequences, annotated genomes (as .gff files) were updated with the Enzyme 527 Commision (EC) numbers, Gene Ontology (GO) terms and predicted function for each protein family 528 group from the pan-genome analysis, using the script gffs2gbk.py in pathwaytools_gff2gbk. This script 529 also appropriately organises the input data required for mpwt given a file listing the taxon IDs for each 530 genome. Pathway tools was then run by running the multi-processing wrapper mpwt was then run with 531 the "--patho" and "--taxon_id" flags, whilst providing the file containing taxon ids linked to each genome. The in silico-reconstructed metabolic pathways for all genomes were then collated using compare_pgdbs.R in pathwaytools_gff2gbk, and downstream analysis conducted in R, as shown in https://github.com/djw533/Serratia_genus_paper/figure_scripts.

\section{Plasmid replicon identification}

536 Plasmid sequences were identified in the collection of Serratia genome assemblies with the MOB-

537 recon tool using the MOB-suite v3.0.3 databases and default settings ${ }^{79}$. Characterisation of the

538 identified plasmids, including predicted transferability of the plasmid, was performed with MOB-

539 typer from the MOB-suite package. Charts illustrating plasmid counts and features were generated in

$540 \mathrm{R}$ using ggplot $2^{80}$. K-mer-based sketches of the plasmid sequences ( $\mathrm{s}=1000, \mathrm{k}=21$ ) were generated

541 with the mash v2.3 sketch algorithm ${ }^{43}$. Pairwise mutation distances between sketches were estimated

542 using mash dist with a distance threshold of 0.05 and otherwise default settings. The resulting all-

543 pairs distance matrix was used for graph-based clustering of the plasmid sequences in Cytoscape

544 v3.8.2 $2^{75}$ using the "connected components cluster" algorithm from the clusterMaker2 v2 app ${ }^{81}$

\section{GC content analysis}

546 Whole-genome GC content calculated using Quast v4.6.0. GC content for each gene, and the average $547 \mathrm{GC}$ value for codon positions 1,2 and 3 for each gene was then calculated using the script 548 GC_from_panaroo_gene_alignments.py, which uses the gene_data.csv file created from Panaroo 
549 (detailed above). Intragenic nucleotide sequence was extracted for all protein-encoding sequences using

550 gals_parser_with_fasta.py with the "-t nuc" flag. Intergenic GC values were then calculated by using

551 Bedtools ${ }^{82}$ complement from Bedtools v2.29.0 to identify the inverse of all coding regions (i.e all

552 intergenic regions). Bedtools getfasta from Bedtools v2.29.0 was then used to extract the intergenic

553 regions as nucleotide sequence. Average GC values for the total intergenic and intragenic regions were

554 then calculated using get_gc_content.py.

\section{Retrieval of specific gene clusters}

556 Gene clusters containing co-localised pig genes (pigA-M) were identified using Hamburger

557 (github.com/djw533/hamburger), which uses protein HMM profiles for each target gene in the gene 558 cluster. User-set parameters define the minimum number of HMMsearch ${ }^{83}$ hits required to report the 559 presence of each system in a genome, in addition to the maximum number of non-hit genes that are 560 permitted between two hit genes in a contiguous set of genes. Gene clusters were reported as prodigiosin 561 clusters for loci encoding at least nine genes containing Pfam domains characteristic of 11 of the 14 pig 562 genes with no more than five non-model genes between any "hits". Extracted genomic sequences were 563 then compared using blast $+\mathrm{v} 2.2 .31^{84}$ and genoplotR v0.8.11 ${ }^{85}$. Blastn was used with the flags "--task 564 Blastn --perc_identity 20 --evalue 10000". Functions created to use these can be found in 565 micro.gen.extra on https://github.com/djw533/micro.gen.extra.

566 Gene clusters around other genes of interest, such as the plasticity zone in S. marcescens located 567 between tRNA-Proggg and tRNA-Ser ${ }_{\text {tga }}$, were extracted using the script pull_out_around_point.py, and, 568 if in the unwanted orientation, flipped using gff_reverse.py.

\section{Phage prediction}

570 Phage regions were predicted using Phaster $^{86}$ on the webserver (https://phaster.ca/), using default 571 settings.

\section{Data visualisation}

573 Phylogenetic trees were visualised using the R package ggtree v2.4.2 ${ }^{88}$. Synteny of regions of bacterial 574 genomes extracted by Hamburger were visualised using the R package genoplotR v0.8.11 ${ }^{85}$. Genetic 575 organisation of genes were plotted using the R package gggenes v0.4.1 (https://wilkox.org/gggenes/). 576 Other plots were created using the R package ggplot2 v3.3.5 $5^{80}$. As mentioned above, networks were 577 viewed using Cytoscape v3.7.175. Sets were visualised as Upset plots using UpsetR v1.4.0 ${ }^{89}$.

\section{Code availability statement}

580 All custom scripts for which github repositories are not specified above can be found in 
https://github.com/djw533/Serratia_genus_paper/, along with all Rscripts used to plot figures. Rscripts make use of the tidyverse ${ }^{87}$ collection of packages. $\mathrm{R}$ version 4.0 .3 was used for all analysis and plotting. Other packages can be found at https:/github.com/djw533/hamburger, https://github.com/djw533/micro.gen.extra, and https://github.com/djw533/pathwaytools_gff2gbk.

585

\section{Data availability statement}

The whole genome sequences generated during the current study are in the process of deposition and will be made fully available. The read sets for the majority of the sequences (from the Institut Pasteur isolates) are already available in the ENA (https://www.ebi.ac.uk/ena/browser/home), with project number PRJEB24638. Other whole genome sequences analysed during the study are available from NCBI GenBank (https://ftp.ncbi.nlm.nih.gov/genomes/genbank/), with the accession numbers for the individual sequences given in Supplementary Table 1.

\section{References}

1. Merlino, C. P. Bartolomeo Bizio's letter to the most eminent priest, Angelo Bellani, concerning the phenomenon of the red-colored polenta [translated from the Italian]. Journal of Bacteriology (1924).

2. Grimont, P. A. D. \& Dulong de Rosnay, H. L. C. Numerical Study of 60 Strains of Serratia. Journal of General Microbiology 72, 259-268 (1972).

3. Grimont, P. A. D., Grimont, F. \& Dulong de Rosnay, H. L. C. Taxonomy of the genus Serratia. Journal of General Microbiology 98, 39-66 (1977).

4. Grimont, F., Grimont, P. A. D. \& Dulong de Rosnay, H. L. C. Characterization of Serratia marcescens, S. liquefaciens, S. plymuthica and S. marinorubra by Electrophoresis of their Proteinases. Journal of General Microbiology 99, 301-310 (1977).

5. Grimont, P. A. D. et al. Deoxyribonucleic Acid Relatedness Between Serratia plymuthica and Other Serratia Species, with a Description of Serratia odorifera sp. nov. (Type Strain: ICPB 3995). International Journal of Systemic Bacteriology 28, 453-463(1978).

6. Grimont, P. A. D., Grimont, F. \& Starr, M. P. Serratia ficaria sp. nov., a bacterial species associated with Smyrna figs and the fig wasp Blastophaga psenes. Current Microbiology 2 , 277-282 (1979).

7. Gavini, F. et al. Serratia fonticola, a New Species from Water International Journal of Systemic Bacteriology 29, 92-101 (1979).

8. Holmes, B. Proposal to Conserve the Specific Epithet liquefaciens Over the Specific Epithet proteamaculans in the Name of the Organism Currently Known as Serratia liquefaciens (Grimes and Hennerty 1931) Bascomb et al. 1971. Request for an Opinion. International Journal of Systemic Bacteriology 30, 220-222 (1980).

9. Grimont, P. A. D., Grimont, F. \& Star, M. P. Serratia species isolated from plants. Current Microbiology 5, 317-322 (1981).

10. Grimont, P. A. D., Grimont, F. \& Irino, K. Biochemical characterization of Serratia liquefaciens sensu stricto, Serratia proteamaculans, and Serratia grimesii sp. nov. Current Microbiology 7 , 69-74 (1982).

11. Grimont, P. A. D., Irino, K. \& Grimont, F. The Serratia liquefaciens-S. proteamaculans-S. grimesii complex: DNA relatedness. Current Microbiology 7, 63-67 (1982).

12. Grimont, P. A. D., Jackson, T. A., Ageron, E. \& Noonan, M. J. Serratia entomophila sp. nov. 
Associated with Amber Disease in the New Zealand Grass Grub Costelytra zealandica. International Journal of Systematic Bacteriology 38, 1-6 (1988).

13. Murdoch, S. L. et al. The opportunistic pathogen Serratia marcescens utilizes Type VI secretion to target bacterial competitors. Journal of Bacteriology 193, 6057-69 (2011).

14. Williamson, N. R., Fineran, P. C., Ogawa, W., Woodley, L. R. \& Salmond, G. P. C. Integrated regulation involving quorum sensing, a two-component system, a GGDEF/EAL domain protein and a post-transcriptional regulator controls swarming and RhlA-dependent surfactant biosynthesis in Serratia. Environmental Microbiology 10, 1202-1217 (2008).

15. Kurz, C. L. et al. Virulence factors of the human opportunistic pathogen Serratia marcescens identified by in vivo screening. The EMBO journal 22, 1451-60 (2003).

16. Khanna, A., Khanna, M. \& Aggarwal, A. Serratia marcescens - a rare opportunistic nosocomial pathogen and measures to limit its spread in hospitalized patients. Journal of Clinical and Diagnostic Research 7, 243-6 (2013).

17. Mahlen, S. D. Serratia infections: from military experiments to current practice. Clinical Microbiology Reviews 24, 755-91 (2011).

18. Moradigaravand, D., Boinett, C. J., Martin, V., Peacock, S. J. \& Parkhill, J. Recent independent emergence of multiple multidrug-resistant Serratia marcescens clones within the United Kingdom and Ireland. Genome Research 26, 1101-1109 (2016).

19. Karkey, A. et al. Outbreaks of Serratia marcescens and Serratia rubidaea bacteremia in a central Kathmandu hospital following the 2015 earthquakes. Transactions of The Royal Society of Tropical Medicine and Hygiene 112, 467-472 (2018).

20. Dubouix, A. et al. Epidemiological investigation of a Serratia liquefaciens outbreak in a neurosurgery department. Journal of Hospital Infection 60, 8-13 (2005).

21. Grimont, F. \& Grimont, P. A. D. The Genus Serratia. in Prokaryotes (eds. Martin Dworkin, Stanley Falkow, Eugene Rosenberg, Karl-Heinz Schleifer \& Erko Stackebrandt) 219-244 (Springer-Verlag, 2006).

22. Hurst, M. R. H., Glare, T. R., Jackson, T. A. \& Ronson, C. W. Plasmid-Located Pathogenicity Determinants of Serratia entomophila, the Causal Agent of Amber Disease of Grass Grub, Show Similarity to the Insecticidal Toxins of Photorhabdus luminescens. Journal of Bacteriology 182, 5127-5138 (2000).

23. Hurst, M. R. H., Glare, T. R. \& Jackson, T. A. Cloning Serratia entomophila antifeeding genes - a putative defective prophage active against the grass grub Costelytra zealandica. Journal of Bacteriology 186, 5116-28 (2004).

24. Nuñez-Valdez, M. E. et al. Identification of a putative Mexican strain of Serratia entomophila pathogenic against root-damaging larvae of Scarabaeidae (Coleoptera). Applied and Environmental Microbiology 74, 802-10 (2008).

25. Rodríguez-Segura, Z., Chen, J., Villalobos, F. J., Gill, S. \& Nuñez-Valdez, M. E. The lipopolysaccharide biosynthesis core of the Mexican pathogenic strain Serratia entomophila is associated with toxicity to larvae of Phyllophaga blanchardi. Journal of Invertebrate Pathology 110, 24-32 (2012).

26. Hurst, M. R. H. et al. Serratia proteamaculans Strain AGR96X Encodes an Antifeeding Prophage (Tailocin) with Activity against Grass Grub (Costelytra giveni) and Manuka Beetle (Pyronota Species) Larvae. Applied and Environmental Microbiology 84, (2018).

27. Flyg, C., Kenne, K. \& Boman, H. G. Insect Pathogenic Properties of Serratia marcescens: Phage-resistant Mutants with a Decreased Resistance to Cecropia Immunity and a Decreased Virulence to Drosophila. Microbiology 120, 173-181 (1980).

28. Ishii, K., Adachi, T., Hara, T., Hamamoto, H. \& Sekimizu, K. Identification of a Serratia marcescens virulence factor that promotes hemolymph bleeding in the silkworm, Bombyx mori. Journal of Invertebrate Pathology 117, 61-67 (2014).

29. Raymann, K., Coon, K. L., Shaffer, Z., Salisbury, S. \& Moran, N. A. Pathogenicity of Serratia marcescens strains in honey bees. mBio 9, e01649-18 (2018).

30. Ashelford, K. E., Fry, J. C., Bailey, M. J. \& Day, M. J. Characterization of Serratia isolates from soil, ecological implications and transfer of Serratia proteamaculans subsp. quinovora Grimont et al. 1983 to Serratia quinivorans corrig., sp. nov. International Journal of Systematic and 
Evolutionary Microbiology 52, 2281-2289 (2002).

31. Lim, Y.-L. L. et al. Complete genome sequence of Serratia fonticola DSM 4576T, a potential plant growth promoting bacterium. Journal of Biotechnology 214, 43-44 (2015).

32. Abebe-Akele, F. et al. Genome sequence and comparative analysis of a putative entomopathogenic Serratia isolated from Caenorhabditis briggsae. BMC Genomics 16, 531 (2015).

33. Petersen, L. M. \& Tisa, L. S. Friend or foe? A review of the mechanisms that drive Serratia towards diverse lifestyles. Canadian Journal of Microbiology 59, 627-640 (2013).

34. Cheng, T. H. et al. Genome Sequence of Serratia marcescens subsp. sakuensis Strain K27, a Marine Bacterium Isolated from Sponge (Haliclona amboinensis). Genome Announcements 6 , e00022-18 (2018).

35. Matilla, M. A., Udaondo, Z. \& Salmond, G. P. C. Genome Sequence of the Oocydin AProducing Rhizobacterium Serratia plymuthica 4Rx5. Microbiology Resource Announcements 7, e00997-18 (2018).

36. Chen, S., Blom, J. \& Walker, E. D. Genomic, Physiologic, and Symbiotic Characterization of Serratia marcescens Strains Isolated from the Mosquito Anopheles stephensi. Frontiers in Microbiology 8, 1483 (2017).

37. Jain, C., Rodriguez-R, L. M., Phillippy, A. M., Konstantinidis, K. T. \& Aluru, S. High throughput ANI analysis of $90 \mathrm{~K}$ prokaryotic genomes reveals clear species boundaries. Nat. Commun. 9, (2018).

38. Horesh, G. et al. Different evolutionary trends form the twilight zone of the bacterial pangenome. Microbial Genomics 7, 000670 (2021).

39. Karp, P. D. et al. Pathway Tools version 23.0 update: software for pathway/genome informatics and systems biology. Briefings in Bioinformatics 22, 109 (2021).

40. Foerstner, K. U., von Mering, C., Hooper, S. D. \& Bork, P. Environments shape the nucleotide composition of genomes. EMBO Reports 6, 1208-1213 (2005).

41. Palidwor, G. A., Perkins, T. J. \& Xia, X. A General Model of Codon Bias Due to GC Mutational Bias. PLOS ONE 5, e13431 (2010).

42. Reuter, S. et al. Parallel independent evolution of pathogenicity within the genus Yersinia. Proceedings of the National Academy of Sciences of the United States of America 111, 67686773 (2014).

43. Ondov, B. D. et al. Mash: Fast genome and metagenome distance estimation using MinHash. Genome Biology 17, 132 (2016).

44. Harris, A. K. P. et al. The Serratia gene cluster encoding biosynthesis of the red antibiotic, prodigiosin, shows species- and strain-dependent genome context variation. Microbiology 150, 3547-3560 (2004).

45. Kwak, Y., Khan, A. R. \& Shin, J.-H. Genome sequence of Serratia nematodiphila DSM 21420T, a symbiotic bacterium from entomopathogenic nematode. Journal of Biotechnology 193, 1-2 (2015).

46. Matilla, M. A., Udaondo, Z., Krell, T. \& Salmond, G. P. C. Genome Sequence of Serratia marcescens MSU97, a Plant-Associated Bacterium That Makes Multiple Antibiotics. Genome Announcements 5, (2017).

47. Cristina, M. L., Sartini, M. \& Spagnolo, A. M. Serratia marcescens infections in neonatal intensive care units (NICUs). International Journal of Environmental Research and Public Health 16, (2019).

48. Daoudi, A., Benaoui, F., el Idrissi Slitine, N., Soraa, N. \& Rabou Maoulainine, F. M. An Outbreak of Serratia marcescens in a Moroccan Neonatal Intensive Care Unit . Advances in Medicine 2018, 1-4 (2018).

49. Moles, L. et al. Serratia marcescens colonization in preterm neonates during their neonatal intensive care unit stay. Antimicrobial Resistance and Infection Control 8, 135 (2019).

50. Martineau, C. et al. Serratia marcescens outbreak in a neonatal intensive care unit: New insights from next-generation sequencing applications. Journal of Clinical Microbiology 56, (2018).

51. Escribano, E. et al. Influence of a Serratia marcescens outbreak on the gut microbiota establishment process in low-weight preterm neonates. PLOS ONE 14, e0216581 (2019). 
52. Montagnani, C. et al. Serratia marcescens outbreak in a neonatal intensive care unit: Crucial role of implementing hand hygiene among external consultants. BMC Infectious Diseases 15, 11 (2015).

53. Hurst, M. R. H., Becher, S. A. \& O'Callaghan, M. Nucleotide sequence of the Serratia entomophila plasmid pADAP and the Serratia proteamaculans pU143 plasmid virulence associated region. Plasmid 65, 32-41 (2011).

54. Wood, D. E. \& Salzberg, S. L. Kraken: Ultrafast metagenomic sequence classification using exact alignments. Genome Biology 15, 1-12 (2014).

55. Lu, J., Breitwieser, F. P., Thielen, P. \& Salzberg, S. L. Bracken: Estimating species abundance in metagenomics data. PeerJ Computer Science 2017, e104 (2017).

56. Li, H. \& Durbin, R. Fast and accurate short read alignment with Burrows-Wheeler transform. Bioinformatics 25, 1754-1760 (2009).

57. Li, H. et al. The Sequence Alignment/Map format and SAMtools. Bioinformatics 25, 20782079 (2009).

58. Gurevich, A., Saveliev, V., Vyahhi, N. \& Tesler, G. QUAST: quality assessment tool for genome assemblies. Bioinformatics 29, 1072-1075 (2013).

59. Zerbino, D. R. \& Birney, E. Velvet: Algorithms for de novo short read assembly using de Bruijn graphs. Genome Research 18, 821-829 (2008).

60. Boetzer, M., Henkel, C. v., Jansen, H. J., Butler, D. \& Pirovano, W. Scaffolding pre-assembled contigs using SSPACE. Bioinformatics 27, 578-579 (2011).

61. Boetzer, M. \& Pirovano, W. Toward almost closed genomes with GapFiller. Genome Biology 13, 1-9 (2012).

62. Bankevich, A. et al. SPAdes: A new genome assembly algorithm and its applications to singlecell sequencing. Journal of Computational Biology 19, 455-477 (2012).

63. Wick, R. R., Judd, L. M., Gorrie, C. L. \& Holt, K. E. Unicycler: Resolving bacterial genome assemblies from short and long sequencing reads. PLOS Computational Biology 13, e1005595 (2017).

64. Koren, S. et al. Canu: Scalable and accurate long-read assembly via adaptive $\kappa$-mer weighting and repeat separation. Genome Research 27, 722-736 (2017).

65. Hunt, M. et al. Circlator: automated circularization of genome assemblies using long sequencing reads. Genome Biology 16, 294 (2015).

66. Seemann, T. Prokka: rapid prokaryotic genome annotation. Bioinformatics 30, 2068-2069 (2014).

67. Tonkin-Hill, G. et al. Producing polished prokaryotic pangenomes with the Panaroo pipeline. Genome Biology 21, 1-21 (2020).

68. Dixon, P. VEGAN, a package of R functions for community ecology. Journal of Vegetation Science 14, 927-930 (2003).

69. Page, A. J. et al. Roary: rapid large-scale prokaryote pan genome analysis. Bioinformatics 31, 3691-3693 (2015).

70. Page, A. J. et al. SNP-sites: rapid efficient extraction of SNPs from multi-FASTA alignments. Microbial Genomics 2, (2016).

71. Nguyen, L. T., Schmidt, H. A., von Haeseler, A. \& Minh, B. Q. IQ-TREE: A Fast and Effective Stochastic Algorithm for Estimating Maximum-Likelihood Phylogenies. Molecular Biology and Evolution 32, 268 (2015).

72. Hoang, D. T., Chernomor, O., von Haeseler, A., Minh, B. Q. \& Vinh, L. S. UFBoot2: Improving the Ultrafast Bootstrap Approximation. Molecular Biology and Evolution 35, 518-522 (2018).

73. Kalyaanamoorthy, S., Minh, B. Q., Wong, T. K. F., von Haeseler, A. \& Jermiin, L. S. ModelFinder: fast model selection for accurate phylogenetic estimates. Nature Methods 14, 587-589 (2017).

74. Jain, C., Rodriguez-R, L. M., Phillippy, A. M., Konstantinidis, K. T. \& Aluru, S. High throughput ANI analysis of $90 \mathrm{~K}$ prokaryotic genomes reveals clear species boundaries. Nature Communications 2018 9:1 9, 1-8 (2018).

75. Shannon, P. et al. Cytoscape: A software Environment for integrated models of biomolecular interaction networks. Genome Research 13, 2498-2504 (2003). 
76. Tonkin-Hill, G., Lees, J. A., Bentley, S. D., Frost, S. D. W. \& Corander, J. Fast hierarchical Bayesian analysis of population structure. Nucleic Acids Research 47, 5539 (2019).

77. Belcour, A. et al. Inferring Biochemical Reactions and Metabolite Structures to Understand Metabolic Pathway Drift. iScience 23, (2020).

78. Huerta-Cepas, J. et al. eggNOG 5.0: a hierarchical, functionally and phylogenetically annotated orthology resource based on 5090 organisms and 2502 viruses. Nucleic Acids Research 47, D309-D314 (2019).

79. Robertson, J., Bessonov, K., Schonfeld, J. \& Nash, J. H. E. Universal whole-sequence-based plasmid typing and its utility to prediction of host range and epidemiological surveillance. Microbial Genomics 6, 1-12 (2020).

80. Wickham, Hadley. Ggplot2 : elegant graphics for data analysis. (Springer, 2009).

81. Morris, J. H. et al. ClusterMaker: A multi-algorithm clustering plugin for Cytoscape. BMC Bioinformatics 12, 1-14 (2011).

82. Quinlan, A. R. \& Hall, I. M. BEDTools: a flexible suite of utilities for comparing genomic features. Bioinformatics 26, 841-842 (2010).

83. Eddy, S. R. Accelerated Profile HMM Searches. PLoS Computational Biology 7, e1002195 (2011).

84. Camacho, C. et al. BLAST+: Architecture and applications. BMC Bioinformatics 10, 421 (2009).

85. Guy, L., Kultima, J. R., Andersson, S. G. E. \& Quackenbush, J. GenoPlotR: comparative gene and genome visualization in R. Bioinformatics 27, 2334-2335 (2011).

86. Arndt, D. et al. PHASTER: a better, faster version of the PHAST phage search tool. Nucleic Acids Research 44, W16 (2016).

87. Wickham, H. et al. Welcome to the Tidyverse. Journal of Open Source Software 4, 1686 (2019).

88. Yu, G., Smith, D. K., Zhu, H., Guan, Y. \& Lam, T. T.-Y. ggtree : an r package for visualization and annotation of phylogenetic trees with their covariates and other associated data. Methods in Ecology and Evolution 8, 28-36 (2017).

89. Conway, J. R., Lex, A. \& Gehlenborg, N. UpSetR: an R package for the visualization of intersecting sets and their properties. Bioinformatics 33, 2938-2940 (2017).

90. Ward, D. V. et al. Metagenomic Sequencing with Strain-Level Resolution Implicates Uropathogenic E. coli in Necrotizing Enterocolitis and Mortality in Preterm Infants. Cell Reports 14, 2912-24 (2016).

91. Roach, D. J. et al. A Year of Infection in the Intensive Care Unit: Prospective Whole Genome Sequencing of Bacterial Clinical Isolates Reveals Cryptic Transmissions and Novel Microbiota. PLoS Genetics 11, e1005413 (2015).

92. Grimont, F. \& Grimont, P. A. D. Genus XXXIV, Serratia. In Bergey's Manual of Systematic Bacteriology, Volume 2 Part B (eds. George Garrity, Don Brenner, Nole Kreig \& James Staley) 799-810 (Springer, 2005).

\section{Acknowledgements}

This work was supported by Wellcome (grant numbers: 104556, Senior Research Fellowship S.J.C.; 220321, Senior Research Fellowship Renewal S.J.C.; 109118, PhD studentship; 206194, N.R.T), the NIHR (NIHR200639, AMR Capital Award to University of Dundee), and Institut Pasteur and INSERM (P.A.D.G. and F.X.W.).

Firstly, we would like to acknowledge the contribution of, and thank, all those colleagues who contributed over many years to the collection of the Serratia isolates forming the Institut Pasteur collection of Patrick Grimont. We also thank Alistair Leanord, Teresa Inkster, James Chalmers, Gillian Orange and Nigel Smith for providing recent isolates of Serratia marcescens from UK hospitals, and 
835 Hazel Auken and George Salmond for sharing isolates reported previously.

836 We thank Sally Kay, Liz McMinn and Florence Juglas for logistical support, the Wellcome Sanger

837 Institute (WSI) sequencing teams for processing these samples, and Christoph Puethe and the WSI

838 Pathogen Informatics team for help with data management. We thank Gal Horesh, Mat Beale and Matt

839 Dorman for expert technical advice and valuable discussions. For the purpose of Open Access, the

840 authors have applied a CC BY public copyright licence to any Author Accepted Manuscript version

841 arising from this submission.

842

843 Author Contributions

844 D.J.W., N.R.T. and S.J.C. conceived the study; D.J.W. performed the bioinformatics analyses, with 845 contributions from A.C.L. and D.C.L; P.A.D.G., F.G. and E.A. performed identification and 846 biochemical characterisation of Serratia isolates in the Institut Pasteur collection; D.J.W., K.P., E.N. 847 and F.X.W. contributed to isolate resuscitation and sequencing; D.J.W., A.J.C., E.H., M.T.G.H., N.R.T. 848 and S.J.C analysed and interpreted results; D.J.W., N.R.T. and S.J.C. wrote the paper with input from 849 the other authors.

850

\section{Competing Financial Interests}

852 The authors declare no competing financial interests. 
bioRxiv preprint doi: https://doi.org/10.1101/2022.01.11.475790; this version posted January 11, 2022. The copyright holder for this preprint (which was not certified by peer review) is the author/funder, who has granted bioRxiv a license to display the preprint in perpetuity. It is made available under aCC-BY 4.0 International license.
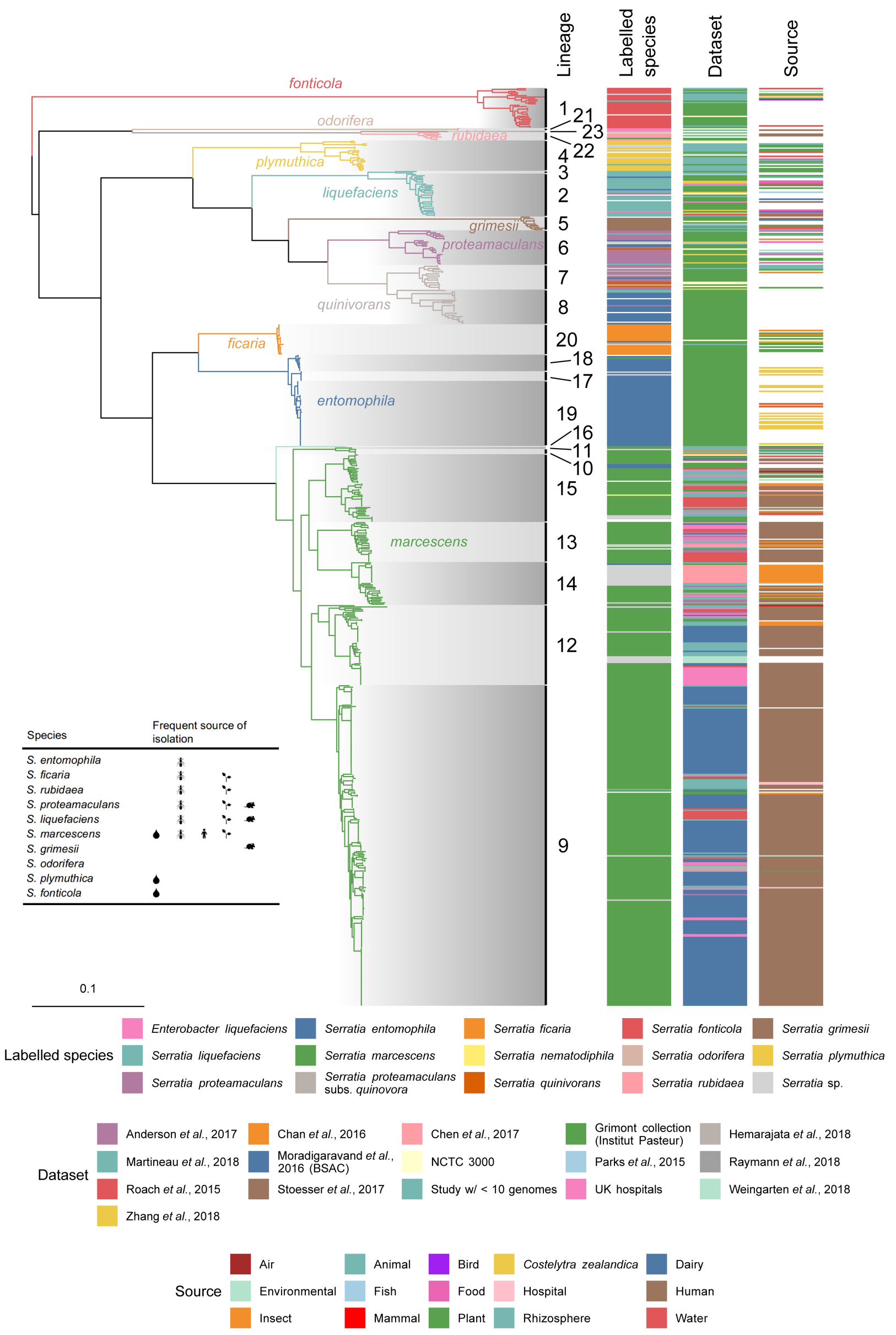

Figure 1: Phylogeny of the genus Serratia. Maximum-likelihood phylogenetic tree constructed from polymorphic sites of a core-gene alignment comprised of 2252 genes from 664 Serratia genomes, comprising 
408 genomes from publicly available databases, and 256 sequenced in this study. Tree constructed with 1000 ultrafast bootstraps. The core-gene alignment was produced from a Panaroo pan-genome analysis run with "-clean_mode moderate" and the protein family threshold set to $70 \%$ shared sequence identity. Branches are coloured according to phylogroups defined by clustering assemblies at $95 \%$ ANI. Clades are shaded according to lineage, calculated through hierarchical bayesian clustering to three levels using FastBaps. "Labelled species" refers to the labelled name of species on the provided Serratia strain sample, or species name associated with published Serratia genome sequences in the NCBI GenBank database. 


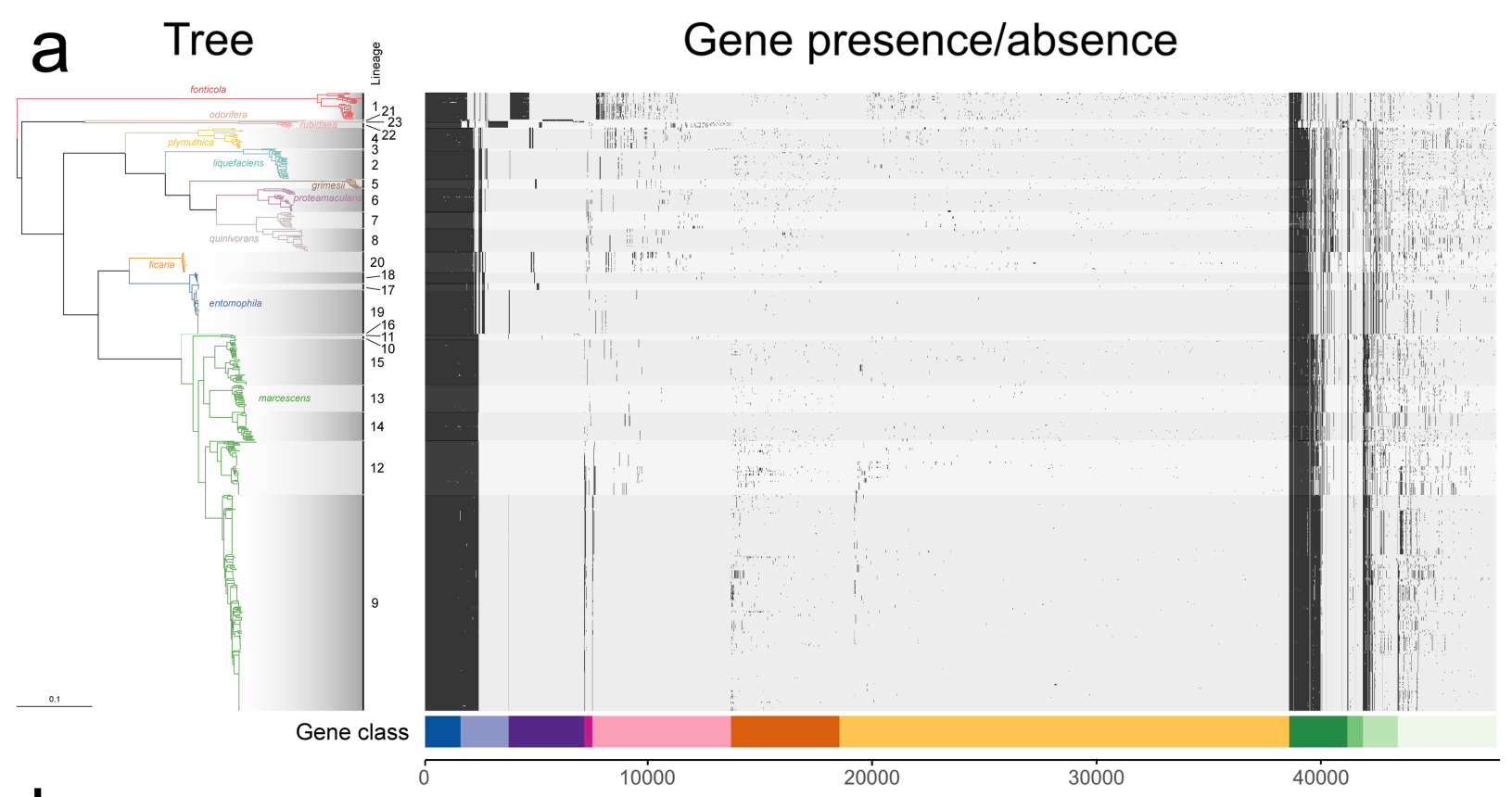

b

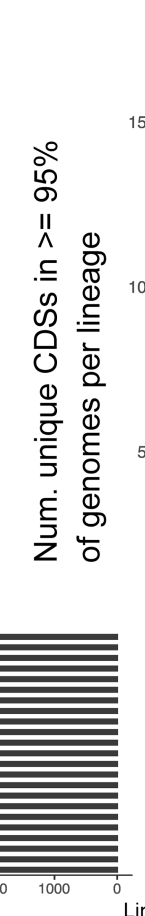

Gene class
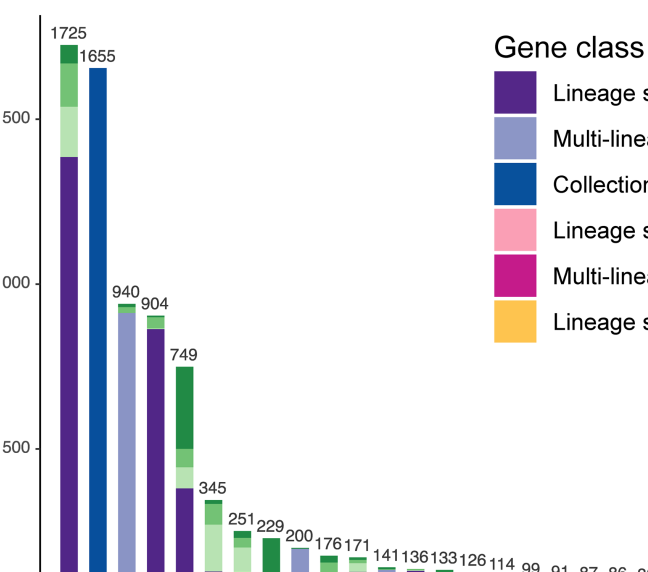

Lineage specific core

Multi-lineage core

Collection core

Lineage specific intermediate

Multi-lineage intermediate

Lineage specific rare

ل1
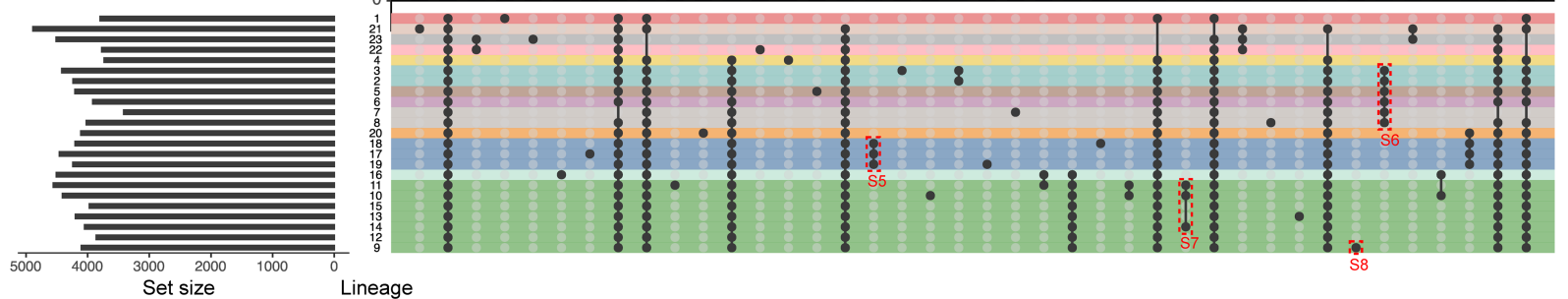

(Size of lineage core genome)

C
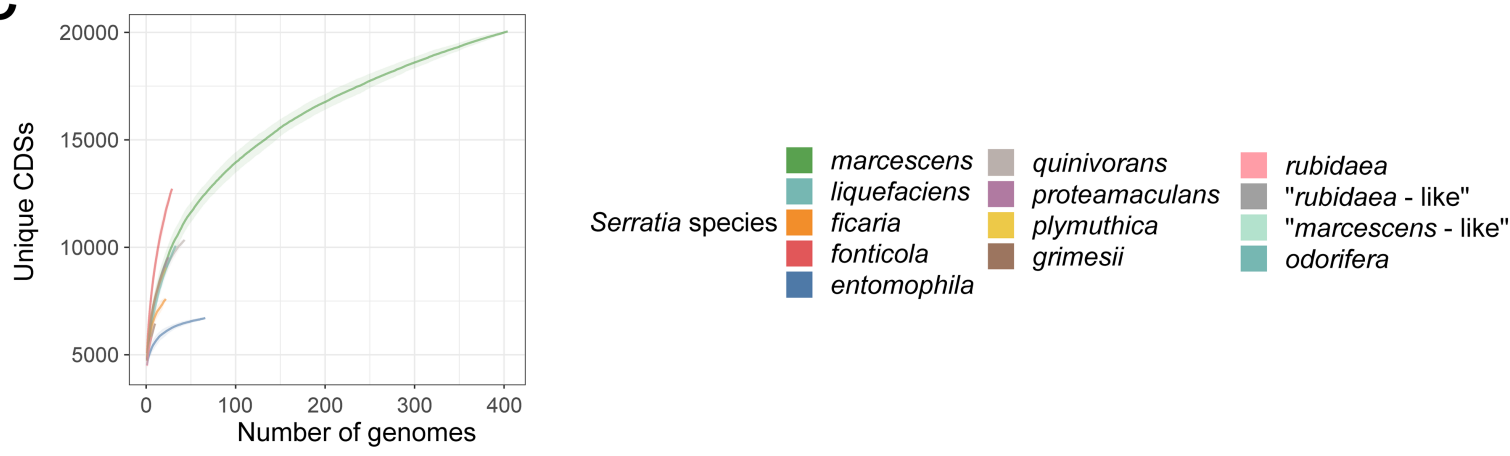

Figure 2: The pan-genome of Serratia. (a) Presence/absence matrix of the 46,588 genes in the Serratia pangenome, generated using Panaroo and overlaid with shading according to lineage, alongside the maximumlikelihood tree based on the core-gene alignment shown in Fig. 1. The presence/absence matrix is ordered by gene class as defined by Twilight. (b) UpSetR plot showing the 50 largest intersections of lineage-specific core 
genomes (genes present in $\geq 95 \%$ of strains in each lineage). Lineages with membership to each intersection are shown by the presence of a black dot in the presence/absence matrix underneath the stacked bar plot. Stacked bar plots representing the number of genes in each intersection are coloured according to the gene classes assigned by Twilight, where singleton lineages have been included (in this case, lineages 22 and 23 are singletons). Rows in the presence/absence matrix correspond to each lineage and are coloured according to Serratia species defined by fastANI. Dashed red boxes indicate intersections of genes represented in Supplementary Figures S5-S8. (c) Estimated pan-genome accumulation curves for each Serratia phylogroup. Shaded region represents standard deviation. 
bioRxiv preprint doi: https://doi.org/10.1101/2022.01.11.475790; this version posted January 11,2022 . The copyright holder for this preprint (which was not certified by peer review) is the author/funder, who has granted bioRxiv a license to display the preprint in perpetuity. It is made available under aCC-BY 4.0 International license.

a

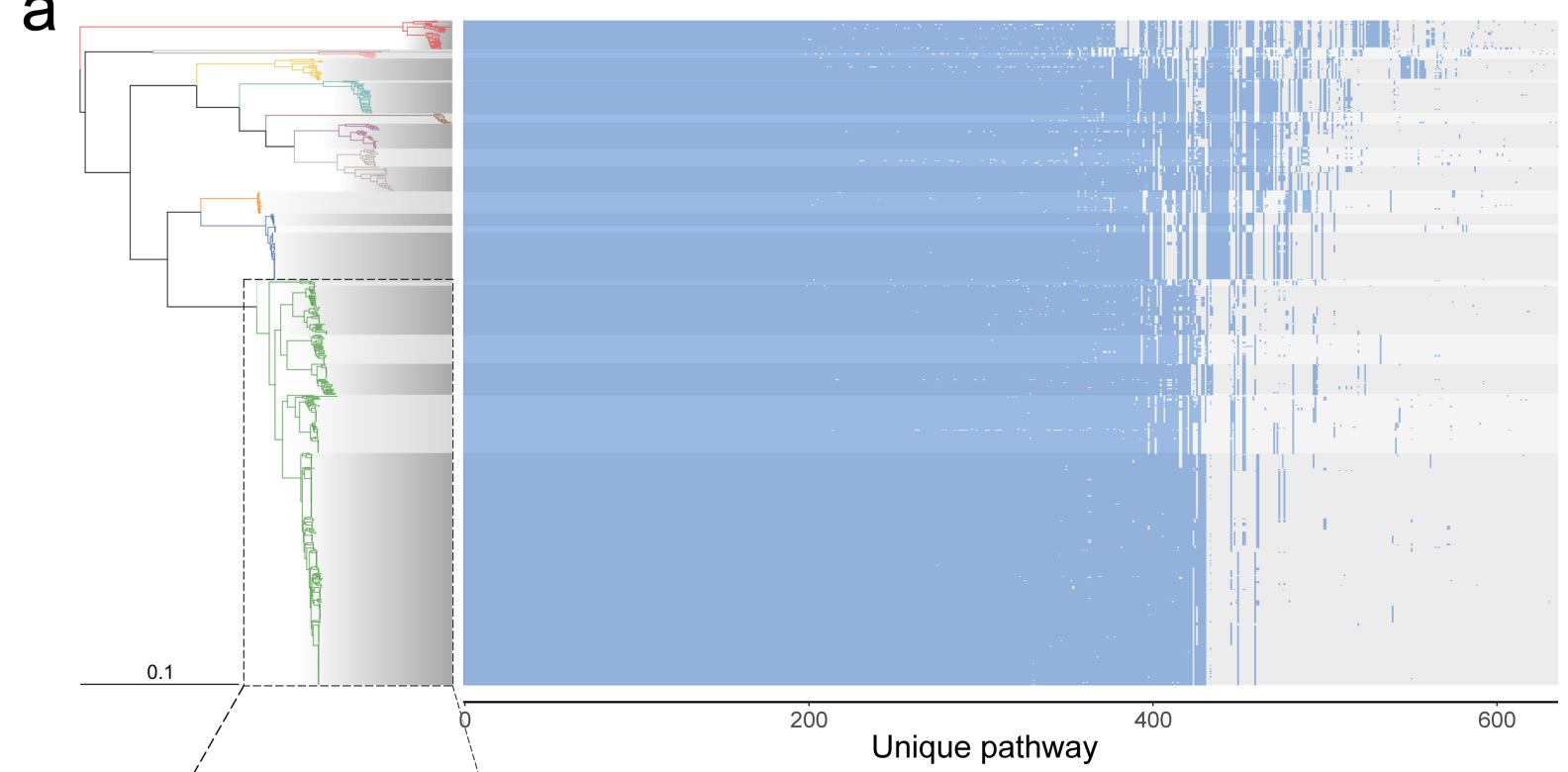

b
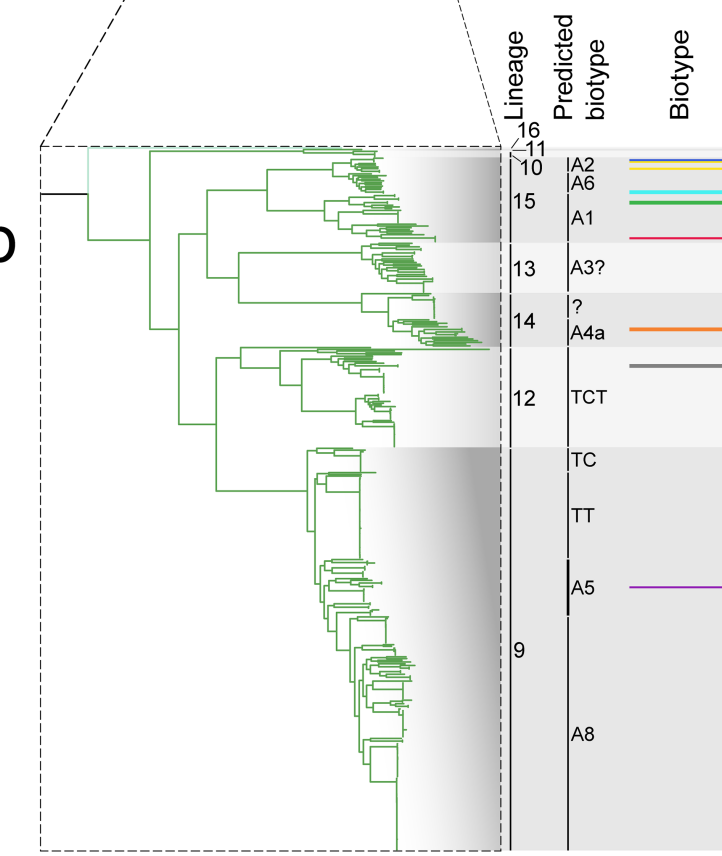

c

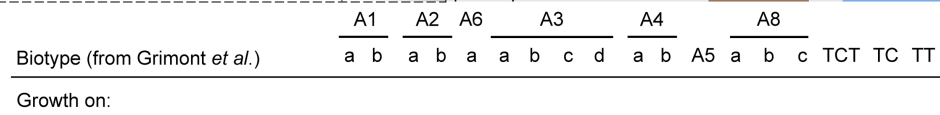
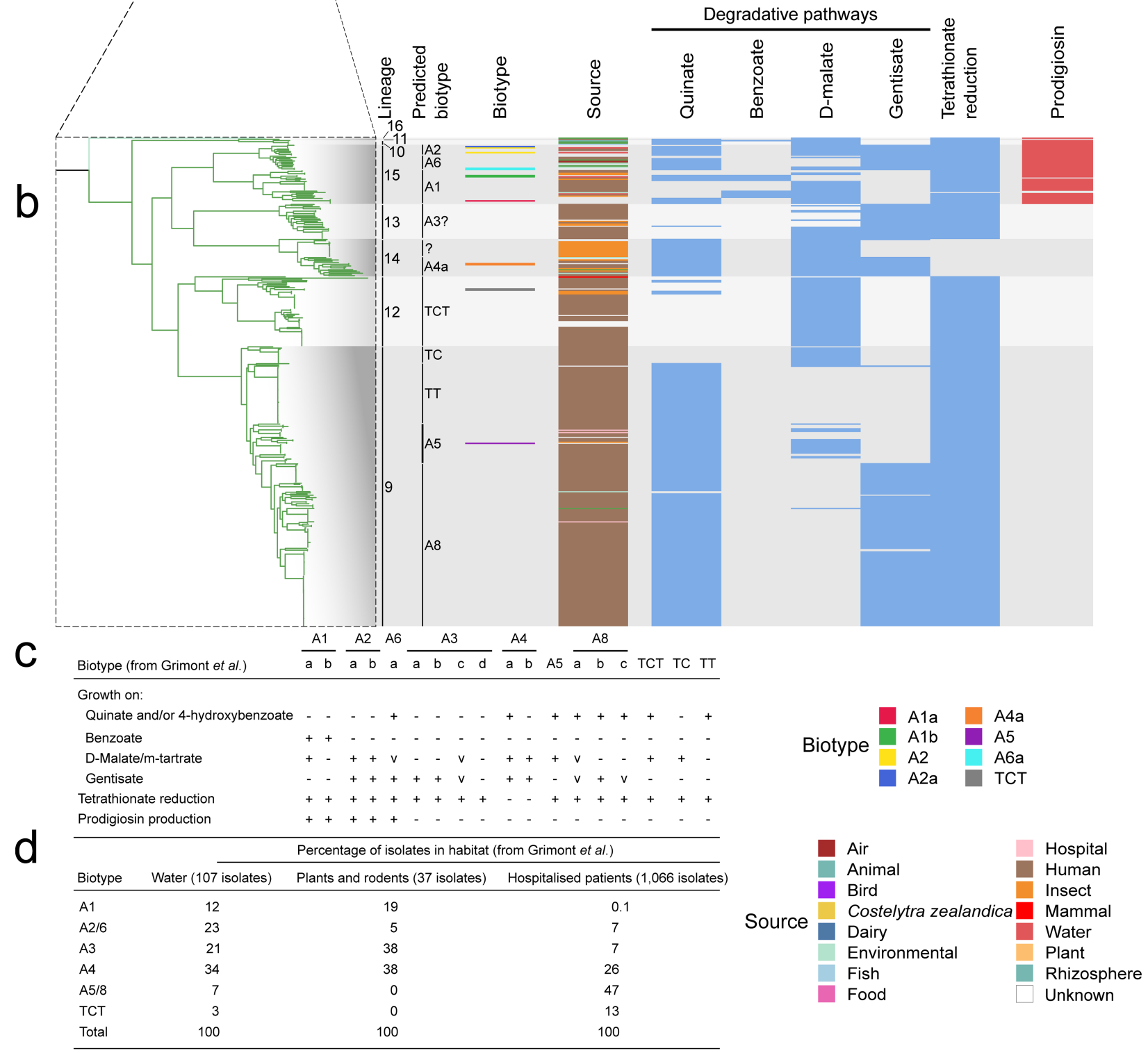

Figure 3: Predicted metabolic pathways in Serratia and correspondence with historical biotyping. (a) Predicted metabolic pathways across Serratia, predicted using Pathway Tools following re-annotation of assemblies using Interproscan/EggNOG-based functional annotation of representative sequences of protein groups defined by Panaroo. (b) Presence/absence of selected complete metabolic pathways across Serratia 
marcescens. Pathways were selected according to a subset of the biochemical tests originally used to group Serratia isolates into Biotypes (c). (d) Table of habitat source for different $S$. marcescens biotypes, reproduced from Grimont et al., 2006. 
bioRxiv preprint doi: https://doi.org/10.1101/2022.01.11.475790; this version posted January 11, 2022. The copyright holder for this preprint (which was not certified by peer review) is the author/funder, who has granted bioRxiv a license to display the preprint in perpetuity. It is made available under aCC-BY 4.0 International license.
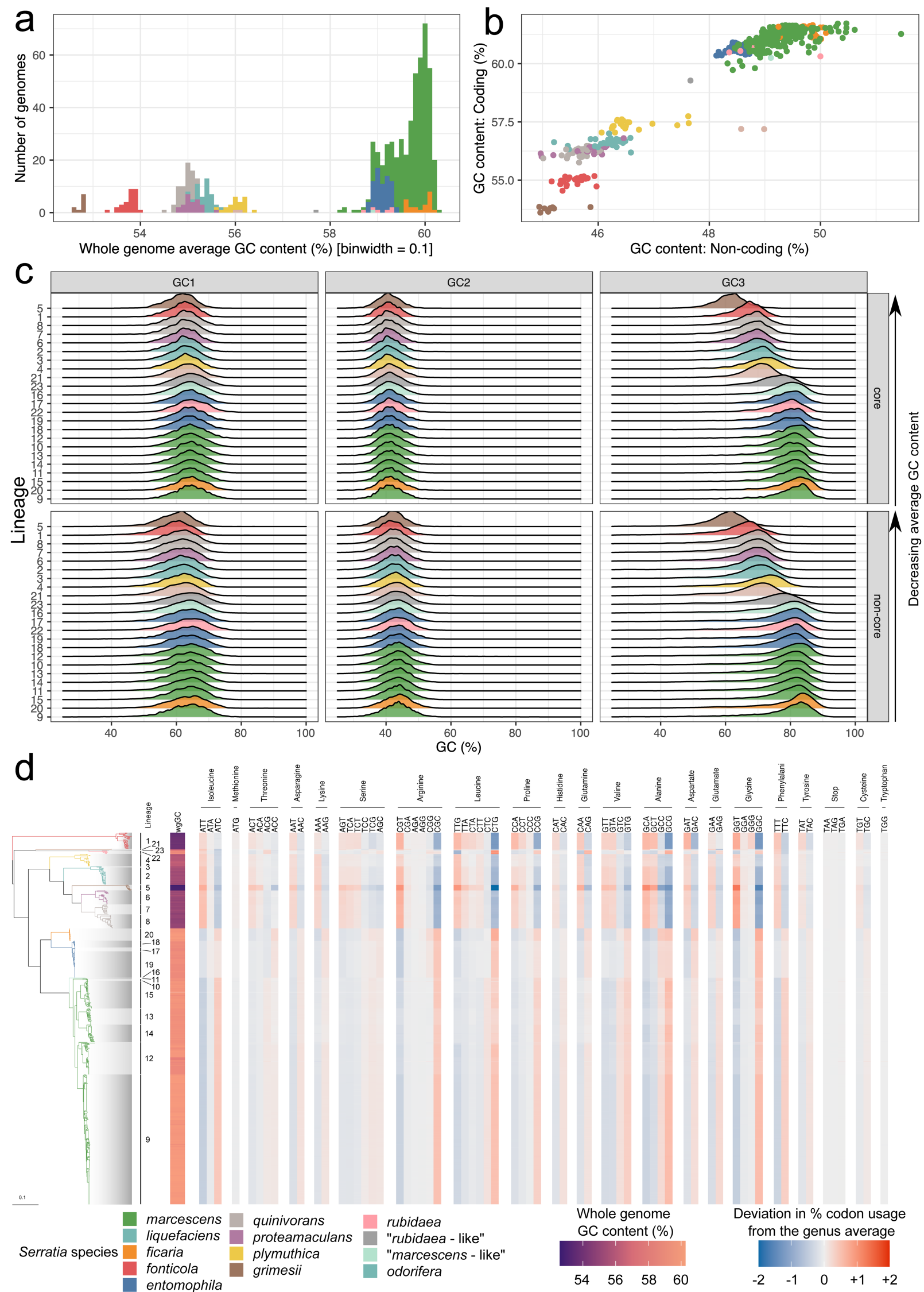

Figure 4: Serratia is split by GC content. (a) Histogram of GC content (average over whole genome) across Serratia. (b) Comparison of GC content in all coding regions vs. that of all intergenic regions. (c) Distribution of GC content in codon positions 1, 2 and 3 in all genus-core (core) and non-genus-core (non-core) genes across each lineage. Data is normalised according to gene length. Ridgeplots are coloured according to Serratia species/phylogroup. Lineages are ordered from top to bottom according to average GC content across the 
whole genome. (d) Codon usage (CU) within the genus-core genome. Blue to red colour represents deviation from the average $\mathrm{CU}$ across the entire genus for each codon, with this genus-average $\mathrm{CU}$ calculated from a per-lineage mean CU value to account for the different numbers of sequences in each lineage. The whole genome GC content is also shown in the left-most column. 


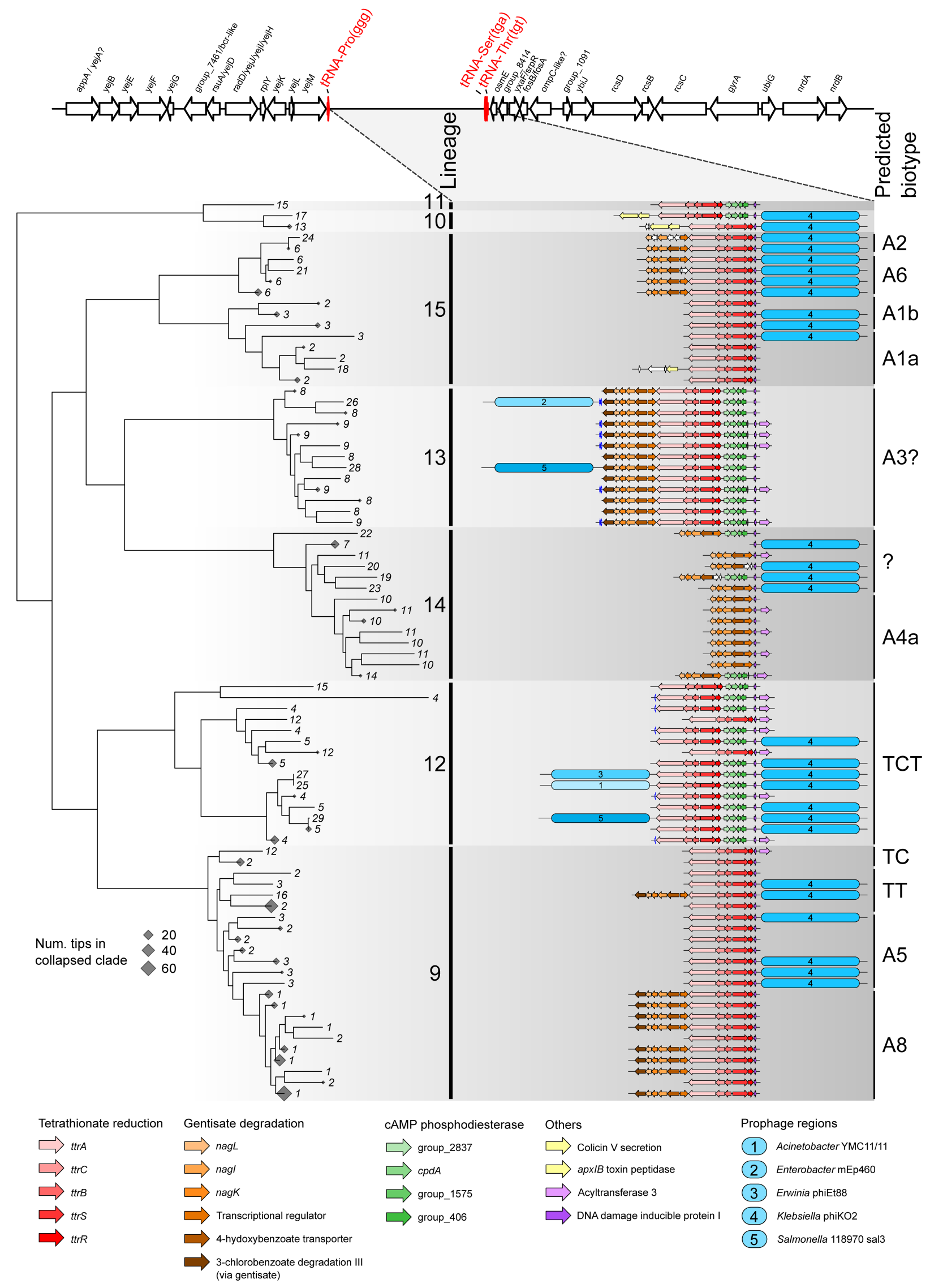

Figure 5: A tRNA-associated hypervariable region ('plasticity zone') is a hot-spot for horizontal transfer of gene cassettes for metabolic pathways used for biotyping within $\boldsymbol{S}$. marcescens. The gene arrangement between the conserved tRNA-Proggg and tRNA-Ser $r_{\text {tga }}$ in $S$. marcescens is plotted against a maximum-likelihood sub-phylogeny from the tree shown in Fig. 1. Clades for which all descending tips represent strains that have an identical set of genes in the locus depicted are collapsed, and denoted by a diamond shape within the tree. 
The size of the diamond represents the number of tips in each collapsed clade. Tips lacking a completely assembled gene locus between tRNA-Proggg and tRNA-Ser tga $_{\text {the }}$ been pruned from the tree. Genes are coloured according to their role, or in the absence of any predicted function, named according to the group number assigned by Panaroo in the pan-genome (Fig. 2). Prophage regions and the closest related prophage sequence determined by PHASTER are indicated. 
bioRxiv preprint doi: https://doi.org/10.1101/2022.01.11.475790; this version posted January 11,2022 . The copyright holder for this preprint (which was not certified by peer review) is the author/funder, who has granted bioRxiv a license to display the preprint in perpetuity. It is made available under aCC-BY 4.0 International license.
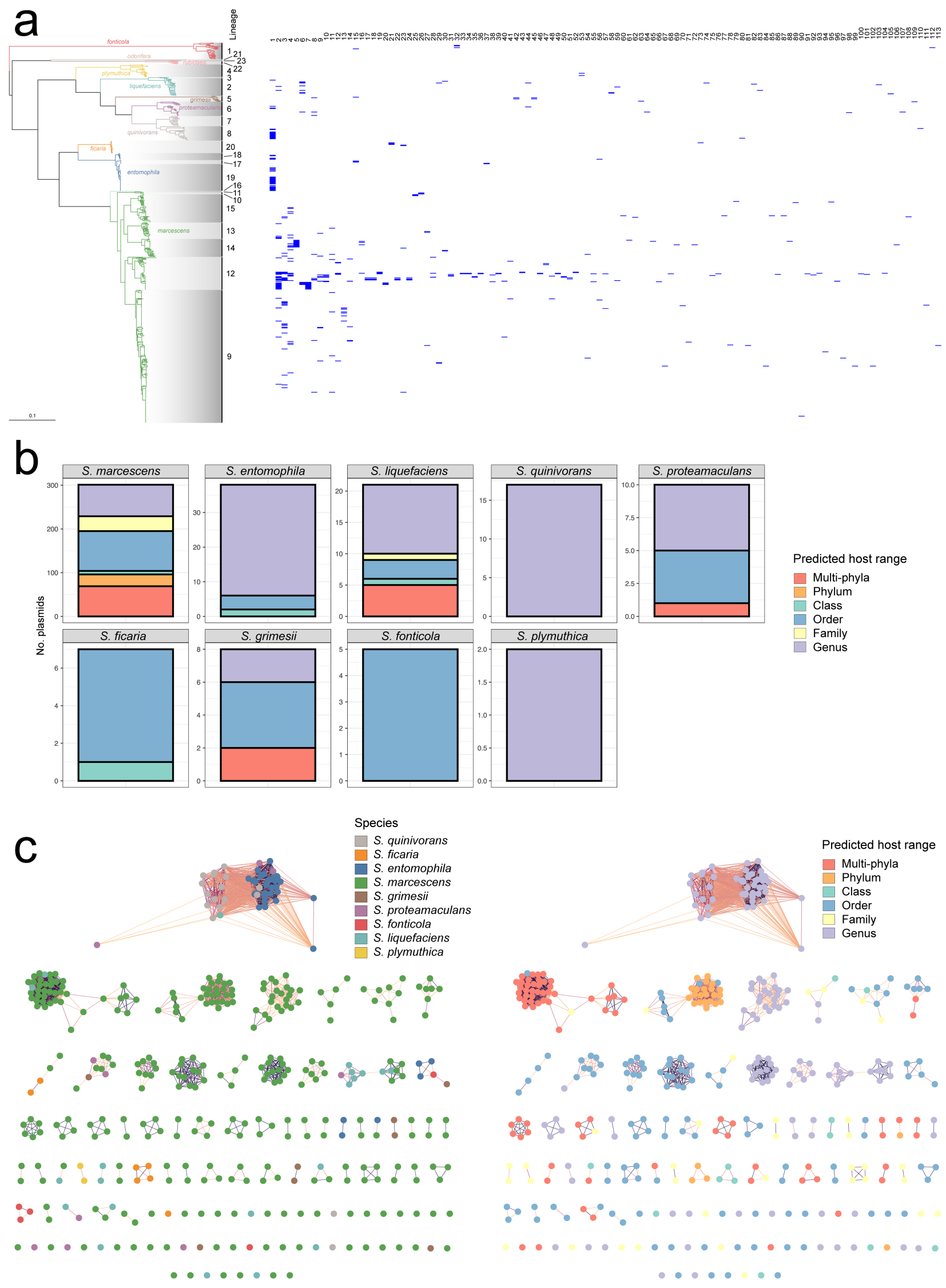

Figure 6: Predicted plasmids across Serratia. (a) Distribution of the 131 plasmid clusters identified against the phylogeny of Serratia shown in Fig. 1. (b) Number and predicted host range of plasmids identified in Serratia genomes. (c) Diversity of Serratia plasmids according to species (left) and predicted host range (right). Within each panel, the order of clusters (from left-right in descending rows) is the same order as presented in the heatmap in panel a (left-right). 


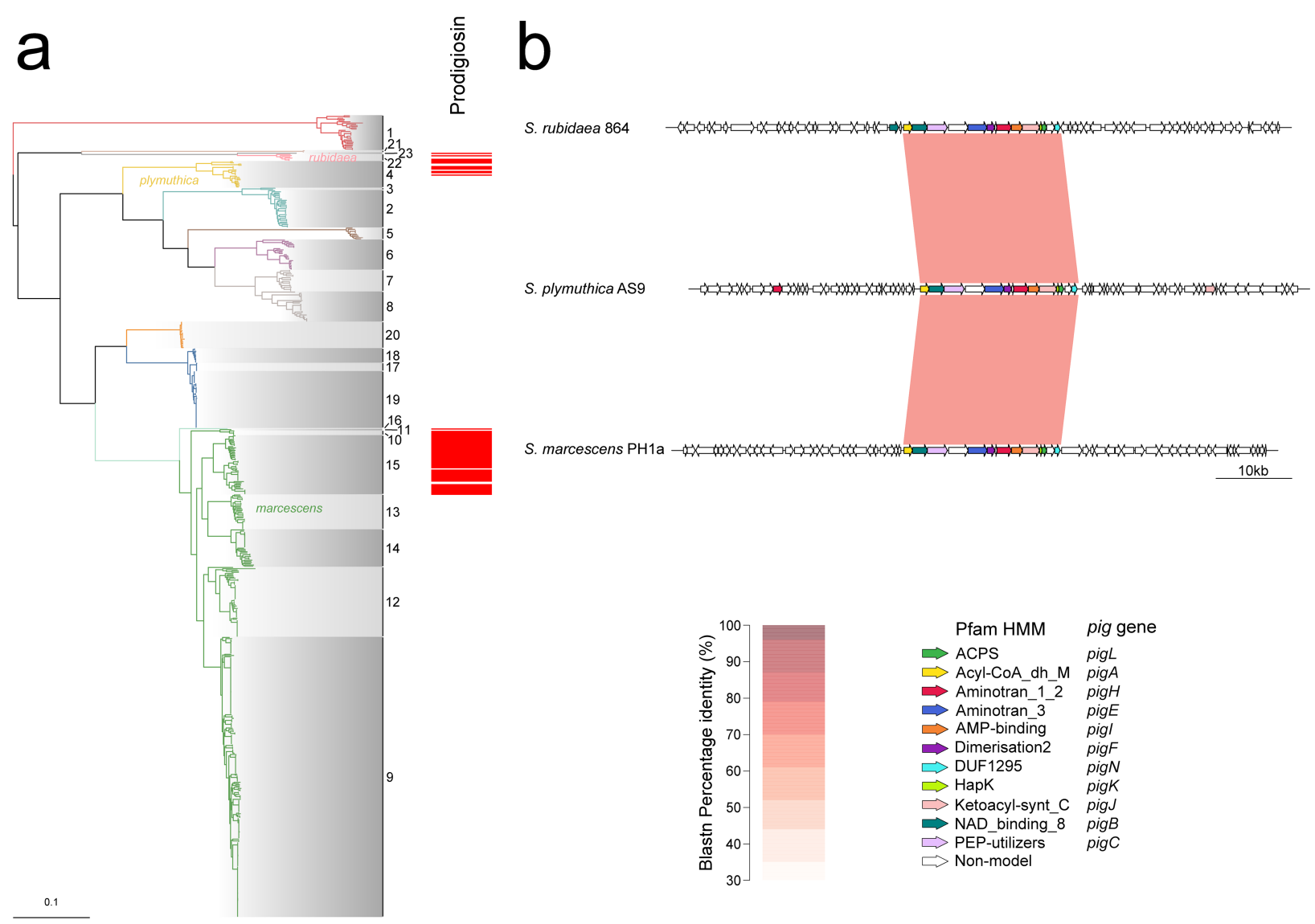

Figure 7: The prodigiosin gene cluster is present variably across Serratia and in different genomic loci. (a) Prodigiosin (pig) gene clusters identified using Hamburger are plotted against the maximum-likelihood phylogeny of Serratia shown in Fig. 1. (b) Pairwise blastn comparison of pig loci (core pig cluster $+/-30 \mathrm{~kb}$ ) from representative members of the three species containing pig genes, extracted using Hamburger. 